\title{
Complexity of Some Generalized Operations on Networks
}

\author{
Muhammad Javaid $\mathbb{1},{ }^{1}$ Hafiz Usman Afzal, ${ }^{1}$ and Shaohui Wang $\mathbb{D}^{2}$ \\ ${ }^{1}$ Department of Mathematics, School of Science, University of Management and Technology, Lahore 54770, Pakistan \\ ${ }^{2}$ Department of Mathematics, Louisiana College, Pineville, LA 71359, USA
}

Correspondence should be addressed to Shaohui Wang; shaohuiwang@yahoo.com

Received 27 March 2021; Accepted 1 July 2021; Published 13 July 2021

Academic Editor: M. De Aguiar

Copyright (c) 2021 Muhammad Javaid et al. This is an open access article distributed under the Creative Commons Attribution License, which permits unrestricted use, distribution, and reproduction in any medium, provided the original work is properly cited.

\begin{abstract}
The number of spanning trees in a network determines the totality of acyclic and connected components present within. This number is termed as complexity of the network. In this article, we address the closed formulae of the complexity of networks' operations such as duplication (split, shadow, and vortex networks of $\left.S_{n}\right)$, sum $\left(S_{n}+W_{3}, S_{n}+K_{2}\right.$, and $\left.\left(C_{n} \circ K_{2}\right)+K_{1}\right)$, product $\left(S_{n} \otimes K_{2}\right.$ and $\left.W_{n} \circ K_{2}\right)$, semitotal networks $\left(Q\left(S_{n}\right)\right.$ and $\left.R\left(S_{n}\right)\right)$, and edge subdivision of the wheel. All our findings in this article have been obtained by applying the methods from linear algebra, matrix theory, and Chebyshev polynomials. Our results shall also be summarized with the help of individual plots and relative comparison at the end of this article.
\end{abstract}

\section{Introduction}

Preliminarily, the finite, connected, and simple network $\Gamma=$ $(V(\Gamma), E(\Gamma))$ shall be our consideration. The complexity (number of spanning trees in a network) is an enormously useful network invariant and is significantly studied in algebraic graph theory, combinatorics, and networking. It admits main connections to computer networking and certain branches of engineering that are related to urban planning (civil engineering) specifically. In fact, the more rigidity and accuracy of a network is ensured by a greater number of spanning trees. Thus, the complexity refers to more perfectness and quality in a network. For further details of its applications, see [1-6].

No general complexity function for an infinite family of networks has been obtained in the literature yet. However, there is a scope of determining closed formulae to find out the complexity of networks of order $n$, especially when the value of $n$ is sufficiently large. With the increase in the order of a network $\Gamma$, it is significant to recapitulate the trend of its complexity function $\tau(\Gamma)$. Among the foremost of such findings, the credit of determining the complexity function of $K_{n}$, as $\tau\left(K_{n}\right)=n^{n-2}$ goes to Cayley [7]. In the same article, another very prominent result proposed by him is the complexity of the complete bipartite network which he enumerated as $\tau\left(K_{m, n}\right)=m^{n-1} n^{m-1}$. The expression for the complexity of Mobius ladder is derived to be $\tau\left(M_{m}\right)=(m / 2)\left[(2+\sqrt{3})^{m}+(2-\sqrt{m})^{m}+2\right]$, for $m \geq 2$ [8].

Recently, determining the number of spanning trees of networks using the determinants of certain matrices has become a hot topic. The most famous among these is Kirchhoff's matrix tree theorem [4]. The aforesaid famous theorem states that the complexity of a network is a cofactor of its Kirchhoff's matrix, where the Kirchhoff's matrix of a network $\Gamma$ is given as $K(\Gamma)=D(\Gamma)-A(\Gamma)$.

The contraction-deletion theorem is a combinatorial technique to enumerate the number of spanning trees of a network. Let $e \in E(\Gamma)$, then the complexity of $\Gamma$ can be calculated iteratively by using

$$
t(\Gamma)=t(\Gamma \mid e)+t(\Gamma-e)
$$

where $\Gamma \mid e$ is obtained by contracting $e=u v$ in $\Gamma$ until the two vertices $u$ and $v$ coincide and $\Gamma-e$ is just the edge deletion with respect to the edge $e$ [9].

The Laplacian spectrum is also helpful for the calculation of the complexity of the network $\Gamma$. Let $\mu_{1}, \mu_{2}, \ldots, \mu_{m}$ be the eigenvalues of the Laplacian matrix $H$ of $\Gamma$. In [10], it is shown that 


$$
\tau(\Gamma)=\frac{1}{m} \prod_{i=1}^{m-1} \mu_{i} .
$$

This method is a complicated one, and complexity of larger networks becomes cumbersome to calculate in this method. Temperley has proven that $\tau(\Gamma)=\left(1 / m^{2}\right)(H+1)$, where 1 is the $m \times m$ matrix with all 1 entries. Some recent work on the enumeration of the complexity of various families of networks can be found in [2, 9, 11-15].

1.1. Definitions and Preliminaries. Temperley's equation mentioned above straightforwardly gives us the following lemma.

Lemma 1 (see [16]). Let $\Gamma$ be an m-order network, then

$$
\tau(\Gamma)=\frac{1}{m^{2}} \operatorname{det}(m I-D(\bar{\Gamma})+A(\bar{\Gamma}))
$$

where $\bar{\Gamma}$ represents the complement of the network $\Gamma$.

The above formula attains significance while enumerating the complexity as it represents $\tau(\Gamma)$ as the determinant of a specific matrix rather than eigenvalues or cofactor.

The first-kind Chebyshev polynomials are defined as the solution of the iterative relation.

$T_{m+1}(x)-2 x T_{m}(x)+T_{m-1}(x)=0, \quad T_{0}(x)=1, T_{1}(x)=x$.

The standard solution of the above iterative relation gives $T_{m}(x)=\frac{1}{2}\left[\left(x+\sqrt{x^{2}-1}\right)^{m}+\left(x-\sqrt{x^{2}-1}\right)^{m}\right], \quad m \geq 1$.

The second-kind Chebyshev Polynomials are defined as the solution of the following iterative relation:

$U_{m+1}(x)-2 x U_{m}(x)+U_{m-1}(x)=0, \quad U_{0}(x)=1, U_{1}(x)=x$.

The standard solution of the above iterative relation gives

$$
\begin{aligned}
U_{m}(x)= & \frac{1}{2 \sqrt{x^{2}-1}} \\
& {\left[\left(x+\sqrt{x^{2}-1}\right)^{m+1}-\left(x-\sqrt{x^{2}-1}\right)^{m+1}\right], \quad m \geq 1 . }
\end{aligned}
$$

Identity (7) is valid for all complex values of $x$ except $x= \pm 1$.

Both first- and second-kind Chebyshev polynomials have a strong link with the determinants [17].
Lemma 2. (see [18, 19]).

(i) $\forall \varphi \geq 3, \operatorname{det}\left[A_{m}(\varphi)\right]=2\left[T_{m}(\varphi / 2)-1\right]$, where

$$
A_{m}(\varphi)=\left(\begin{array}{ccccccccc}
\varphi & -1 & 0 & 0 & \ldots & 0 & 0 & 0 & -1 \\
-1 & \varphi & -1 & 0 & \ldots & 0 & 0 & 0 & 0 \\
0 & -1 & \varphi & -1 & \ldots & 0 & 0 & 0 & 0 \\
0 & 0 & -1 & \varphi & \ldots & 0 & 0 & 0 & 0 \\
\vdots & \vdots & \vdots & \vdots & \ddots & \vdots & \vdots & \vdots & \vdots \\
0 & 0 & 0 & 0 & \ldots & \varphi & -1 & 0 & 0 \\
0 & 0 & 0 & 0 & \ldots & -1 & \varphi & -1 & 0 \\
0 & 0 & 0 & 0 & \ldots & 0 & -1 & \varphi & -1 \\
-1 & 0 & 0 & 0 & \ldots & 0 & 0 & -1 & \varphi
\end{array}\right) .
$$

(ii) $\forall \varphi \geq 4, \quad m \geq 3, \quad \operatorname{det}\left[B_{m}(\varphi)\right]=(2(\varphi+m-3) /$ $(\varphi-3))\left[T_{m}(\varphi-1 / 2)-1\right]$, where

$$
B_{m}(\varphi)=\left(\begin{array}{ccccccccc}
\varphi & 0 & 1 & 1 & \ldots & 1 & 1 & 1 & 0 \\
0 & \varphi & 0 & 1 & \ldots & 1 & 1 & 1 & 1 \\
1 & 0 & \varphi & 0 & \ldots & 1 & 1 & 1 & 1 \\
1 & 1 & 0 & \varphi & \ldots & 1 & 1 & 1 & 1 \\
\vdots & \vdots & \vdots & \vdots & \ddots & \vdots & \vdots & \vdots & \vdots \\
1 & 1 & 1 & 1 & \ldots & \varphi & 0 & 1 & 1 \\
1 & 1 & 1 & 1 & \ldots & 0 & \varphi & 0 & 1 \\
1 & 1 & 1 & 1 & \ldots & 1 & 0 & \varphi & 0 \\
0 & 1 & 1 & 1 & \ldots & 1 & 1 & 0 & \varphi
\end{array}\right) .
$$

(iii) $\forall m, \varphi ; \operatorname{det}\left[C_{m}(\varphi)\right]=(\varphi-1) U_{m-1}(\varphi+1 / 2)$, where

$$
C_{m}(\varphi)=\left(\begin{array}{ccccccccc}
\varphi & -1 & 0 & 0 & \ldots & 0 & 0 & 0 & 0 \\
-1 & \varphi+1 & -1 & 0 & \ldots & 0 & 0 & 0 & 0 \\
0 & -1 & \varphi+1 & -1 & \ldots & 0 & 0 & 0 & 0 \\
0 & 0 & -1 & \varphi+1 & \ldots & 0 & 0 & 0 & 0 \\
\vdots & \vdots & \vdots & \vdots & \ddots & \vdots & \vdots & \vdots & \vdots \\
0 & 0 & 0 & 0 & \ldots & \varphi+1 & -1 & 0 & 0 \\
0 & 0 & 0 & 0 & \ldots & -1 & \varphi+1 & -1 & 0 \\
0 & 0 & 0 & 0 & \ldots & 0 & -1 & \varphi+1 & -1 \\
0 & 0 & 0 & 0 & \ldots & 0 & 0 & -1 & \varphi
\end{array}\right) .
$$

(iv) $\forall \varphi \geq 2, \quad m \geq 3, \quad \operatorname{det}\left[D_{m}(\varphi)\right]=(m+\varphi-2) \quad U_{m-1}$ $(\varphi / 2)$, where 


$$
D_{m}(\varphi)=\left(\begin{array}{ccccccccc}
\varphi & 0 & 1 & 1 & \ldots & 1 & 1 & 1 & 1 \\
0 & \varphi+1 & 0 & 1 & \ldots & 1 & 1 & 1 & 1 \\
1 & 0 & \varphi+1 & 0 & \ldots & 1 & 1 & 1 & 1 \\
1 & 1 & 0 & \varphi+1 & \ldots & 1 & 1 & 1 & 1 \\
\vdots & \vdots & \vdots & \vdots & \ddots & \vdots & \vdots & \vdots & \vdots \\
1 & 1 & 1 & 1 & \ldots & \varphi+1 & 0 & 1 & 1 \\
1 & 1 & 1 & 1 & \ldots & 0 & \varphi+1 & 0 & 1 \\
1 & 1 & 1 & 1 & \ldots & 1 & 0 & \varphi+1 & 0 \\
1 & 1 & 1 & 1 & \ldots & 1 & 1 & 0 & \varphi
\end{array}\right) .
$$

Lemma 3 (see [20]). $\forall \varphi$ and $m, \operatorname{det}\left[W_{m}(\varphi)\right]=$ $(\varphi+m-1)(\varphi-1)^{m-1}$, where $W_{m}(\varphi)$ is an $m \times m$ circulant matrix given as

$$
W_{m}(\varphi)=\left(\begin{array}{ccccccccc}
\varphi & 1 & 1 & 1 & \ldots & 1 & 1 & 1 & 1 \\
1 & \varphi & 1 & 1 & \ldots & 1 & 1 & 1 & 1 \\
1 & 1 & \varphi & 1 & \ldots & 1 & 1 & 1 & 1 \\
1 & 1 & 1 & \varphi & \ldots & 1 & 1 & 1 & 1 \\
\vdots & \vdots & \vdots & \vdots & \ddots & \vdots & \vdots & \vdots & \vdots \\
1 & 1 & 1 & 1 & \ldots & \varphi & 1 & 1 & 1 \\
1 & 1 & 1 & 1 & \ldots & 1 & \varphi & 1 & 1 \\
1 & 1 & 1 & 1 & \ldots & 1 & 1 & \varphi & 1 \\
1 & 1 & 1 & 1 & \ldots & 1 & 1 & 1 & \varphi
\end{array}\right) .
$$

Lemma 4 (see [21]). Let $P, Q, R$, and $S$ be the block matrices of orders $\alpha \times \alpha, \alpha \times \beta, \beta \times \alpha$, and $\beta \times \beta$, respectively. Then,

$$
\begin{aligned}
\operatorname{det}\left(\begin{array}{ll}
P & Q \\
R & S
\end{array}\right) & =\operatorname{det}(P) \times \operatorname{det}\left(S-R P^{-1} Q\right) \\
& =\operatorname{det}(S) \times \operatorname{det}\left(P-Q S^{-1} R\right),
\end{aligned}
$$

where $P$ and $Q$ are nonsingular matrices.

Throughout the article, $A_{m \times n}$ will represent the matrix of order $m \times n . R_{i}$ and $C_{i}$ will represent the $i^{\text {th }}$ row and $i^{\text {th }}$ column in a matrix, respectively. Also, the set of determinant operations $\left({ }^{*}\right)$ performed in Theorem 1 shall be used frequently in all our results.

\section{Main Results}

The realm of generating new structures by applying general operations to the existing ones always remains open in networking. In this section, we shall present our main findings consisting of the enumerated closed formulae of the generalized operation on certain networks. The necessary definition of a network's operation [22,23] shall be provided before the respective result.

Theorem 1. For all $n$, the complexity of the network $S_{n}+W_{3}$ is given by

$$
\tau\left(S_{n}+W_{3}\right)=5^{n-1}(n+5)^{4} .
$$

Proof. Consider the network $S_{n}+W_{3}$ with $\left|V\left(S_{n}+W_{3}\right)\right|=$ $n+5$ and $\left|E\left(S_{n}+W_{3}\right)\right|=5 n+10$, see Figure 1.

Applying Lemma 1, we have 


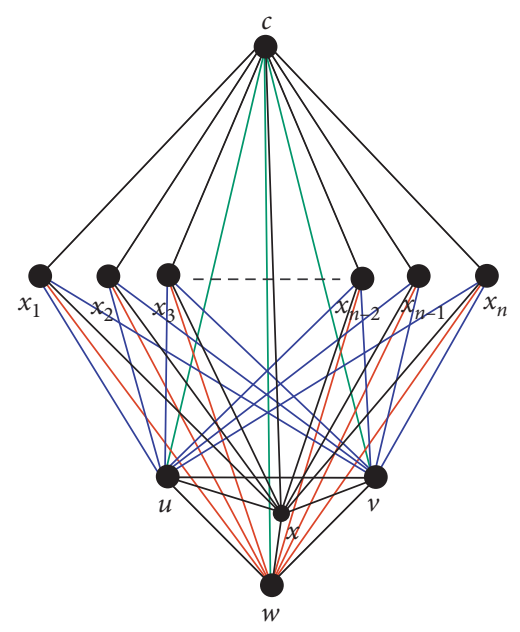

Figure 1: The network $S_{n}+W_{3}$.

$\tau\left(S_{n}+W_{3}\right)=\frac{1}{(n+5)^{2}} \operatorname{det}[(n+5) I-\bar{D}+\bar{A}]$

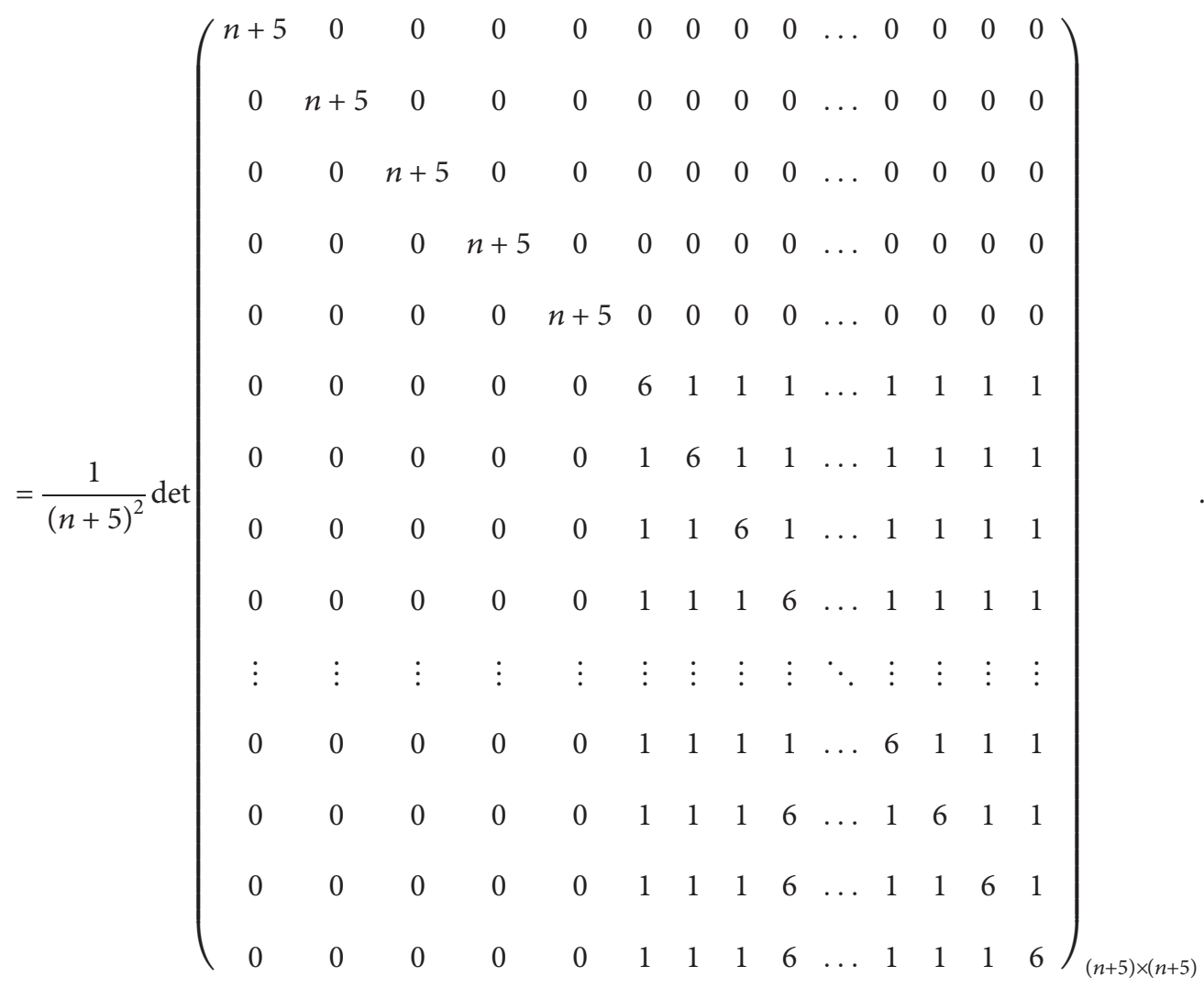

Now, we perform the following operations simultaneously on the above determinant:

(i) Adding all columns to $C_{1}$ (ii) Taking $n+5 \quad$ common from $C_{1}$. $-------\rightarrow(*)$

(iii) Subtracting $C_{1}$ from all columns 
(iv) Expanding along $R_{1}$

These operations yield

$$
=\left(\begin{array}{ccccccccccccc}
n+4 & -1 & -1 & -1 & -1 & -1 & -1 & -1 & \ldots & -1 & -1 & -1 & -1 \\
-1 & n+4 & -1 & -1 & -1 & -1 & -1 & -1 & \ldots & -1 & -1 & -1 & -1 \\
-1 & -1 & n+4 & -1 & -1 & -1 & -1 & -1 & \ldots & -1 & -1 & -1 & -1 \\
-1 & -1 & -1 & n+4 & -1 & -1 & -1 & -1 & \ldots & -1 & -1 & -1 & -1 \\
-1 & -1 & -1 & -1 & 5 & 0 & 0 & 0 & \ldots & 0 & 0 & 0 & 0 \\
-1 & -1 & -1 & -1 & 0 & 5 & 0 & 0 & \ldots & 0 & 0 & 0 & 0 \\
-1 & -1 & -1 & -1 & 0 & 0 & 5 & 0 & \ldots & 0 & 0 & 0 & 0 \\
-1 & -1 & -1 & -1 & 0 & 0 & 0 & 5 & \ldots & 0 & 0 & 0 & 0 \\
\vdots & \vdots & \vdots & \vdots & \vdots & \vdots & \vdots & \vdots & \ddots & \vdots & \vdots & \vdots & \vdots \\
-1 & -1 & -1 & -1 & 0 & 0 & 0 & 0 & \ldots & 5 & 0 & 0 & 0 \\
-1 & -1 & -1 & -1 & 0 & 0 & 0 & 0 & \ldots & 0 & 5 & 0 & 0 \\
-1 & -1 & -1 & -1 & 0 & 0 & 0 & 0 & \ldots & 0 & 0 & 5 & 0 \\
-1 & -1 & -1 & -1 & 5 & 0 & 0 & 0 & \ldots & 0 & 0 & 0 & 5
\end{array}\right)_{(n+4) \times(n+4)}
$$

By using Lemma 4, we obtain

$$
\begin{aligned}
& \tau\left(S_{n}+W_{3}\right)=\operatorname{det}(S) \cdot \operatorname{det}\left(P-Q S^{-1} R\right) \\
& =5^{n} \operatorname{det}\left(\begin{array}{llll}
\frac{4 n+20}{5} & \frac{-(n+5)}{5} & \frac{-(n+5)}{5} & \frac{-(n+5)}{5} \\
\frac{-(n+5)}{5} & \frac{4 n+20}{5} & \frac{-(n+5)}{5} & \frac{-(n+5)}{5} \\
\frac{-(n+5)}{5} & \frac{-(n+5)}{5} & \frac{4 n+20}{5} & \frac{-(n+5)}{5} \\
\frac{-(n+5)}{5} & \frac{-(n+5)}{5} & \frac{-(n+5)}{5} & \frac{4 n+20}{5}
\end{array}\right) \\
& =5^{n}\left(\frac{n+5}{5}\right)^{4}\left(\begin{array}{cccc}
\frac{4 n+20}{-(n+5)} & 1 & 1 & 1 \\
1 & \frac{4 n+20}{-(n+5)} & 1 & 1 \\
1 & 1 & \frac{4 n+20}{-(n+5)} & 1 \\
1 & 1 & 1 & \frac{4 n+20}{-(n+5)}
\end{array}\right) \text {. }
\end{aligned}
$$

Using Lemma 3, we have

$$
\begin{aligned}
\tau\left(S_{n}+W_{3}\right) & =5^{n-4}(n+5)^{4}\left[\frac{4 n+20}{-(n+5)}+3\right]\left[\frac{4 n+20}{-(n+5)}-1\right]^{3} \\
& \Rightarrow \tau\left(S_{n}+W_{3}\right)=5^{n-1}(n+5)^{4} .
\end{aligned}
$$

Definition 1. The shadow $D_{2}\left(\Gamma_{1}\right)$ of a network $\Gamma_{1}$ is obtained by taking another copy of $\Gamma_{1}$, say $\Gamma_{2}$, and then by making all those vertices $u_{i} \in V\left(\Gamma_{1}\right)$ adjacent to the corresponding adjacent vertices $v_{i} \in V\left(\Gamma_{2}\right)$.

Theorem 2. For all $n$, the complexity of the network $D_{2}\left(S_{n}\right)$ is given by

$$
\tau\left(D_{2}\left(S_{n}\right)\right)=n .2^{2 n}
$$

Proof. Consider the network $D_{2}\left(S_{n}\right)$ with $\left|V\left(D_{2}\left(S_{n}\right)\right)\right|=$ $2 n+2$ and $\left|E\left(D_{2}\left(S_{n}\right)\right)\right|=4 n$, see Figure 2 .

Applying Lemma 1, we have

$$
\tau\left(D_{2}\left(S_{n}\right)\right)=\frac{1}{(2 n+2)^{2}} \operatorname{det}[(2 n+2) I-\bar{D}+\bar{A}],
$$

where $\bar{D}$ and $\bar{A}$ represent the degree and adjacency matrices of the network $D_{2}\left(S_{n}\right)$, respectively. 


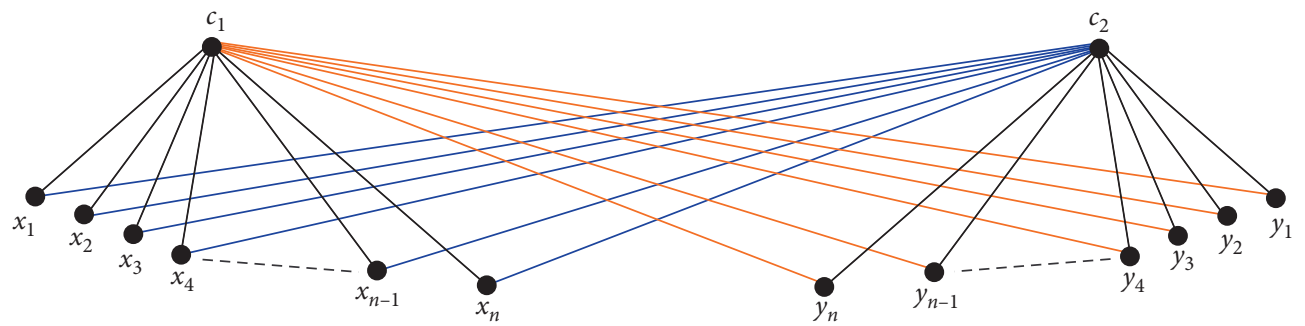

Figure 2: The shadow network $D_{2}\left(S_{n}\right)$.

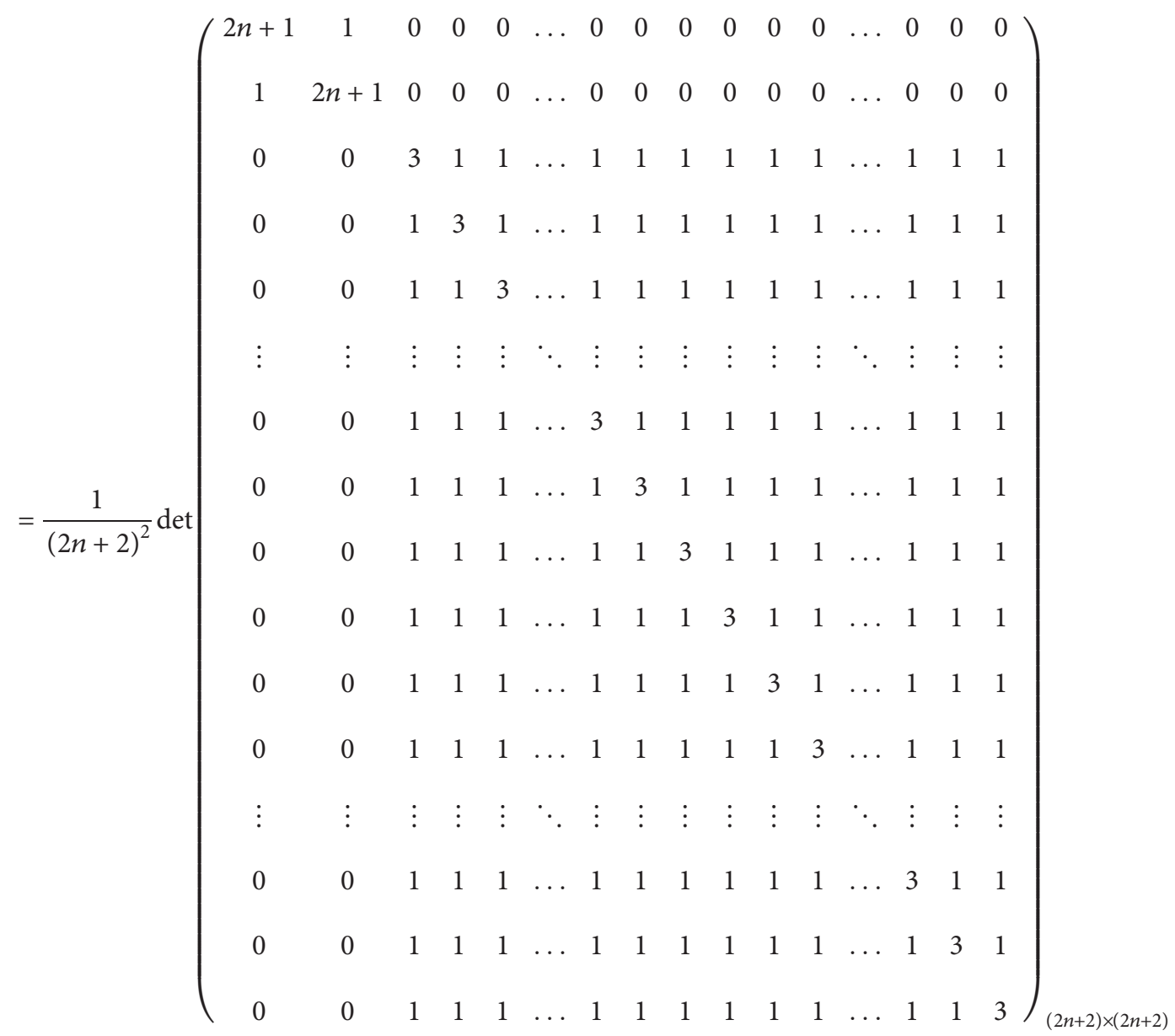




$$
\begin{gathered}
=\operatorname{det}\left(\begin{array}{ccccccccccccccc}
2 n & -1 & -1 & -1 & \ldots & -1 & -1 & -1 & -1 & -1 & -1 & \ldots & -1 & -1 & -1 \\
-1 & 2 & 0 & 0 & \ldots & 0 & 0 & 0 & 0 & 0 & 0 & \ldots & 0 & 0 & 0 \\
-1 & 0 & 2 & 0 & \ldots & 0 & 0 & 0 & 0 & 0 & 0 & \ldots & 0 & 0 & 0 \\
-1 & 0 & 0 & 2 & \ldots & 0 & 0 & 0 & 0 & 0 & 0 & \ldots & 0 & 0 & 0 \\
\vdots & \vdots & \vdots & \vdots & \ddots & \vdots & \vdots & \vdots & \vdots & \vdots & \vdots & \ddots & \vdots & \vdots & \vdots \\
-1 & 0 & 0 & 0 & \ldots & 2 & 0 & 0 & 0 & 0 & 0 & \ldots & 0 & 0 & 0 \\
-1 & 0 & 0 & 0 & \ldots & 0 & 2 & 0 & 0 & 0 & 0 & \ldots & 0 & 0 & 0 \\
-1 & 0 & 0 & 0 & \ldots & 0 & 0 & 2 & 0 & 0 & 0 & \ldots & 0 & 0 & 0 \\
-1 & 0 & 0 & 0 & \ldots & 0 & 0 & 0 & 2 & 0 & 0 & \ldots & 0 & 0 & 0 \\
-1 & 0 & 0 & 0 & \ldots & 0 & 0 & 0 & 0 & 2 & 0 & \ldots & 0 & 0 & 0 \\
-1 & 0 & 0 & 0 & \ldots & 0 & 0 & 0 & 0 & 0 & 2 & \ldots & 0 & 0 & 0 \\
\vdots & \vdots & \vdots & \vdots & \ddots & \vdots & \vdots & \vdots & \vdots & \vdots & \vdots & \ddots & \vdots & \vdots & \vdots \\
-1 & 0 & 0 & 0 & \ldots & 0 & 0 & 0 & 0 & 0 & 0 & \ldots & 2 & 0 & 0 \\
-1 & 0 & 0 & 0 & \ldots & 0 & 0 & 0 & 0 & 0 & 0 & \ldots & 0 & 2 & 0 \\
-1 & 0 & 0 & 0 & \ldots & 0 & 0 & 0 & 0 & 0 & 0 & \ldots & 0 & 0 & 2
\end{array}\right)_{(2 n+1) \times(2 n+1)} \\
\Rightarrow \tau\left(D_{2}\left(S_{n}\right)\right)
\end{gathered}
$$

By using Lemma 4, we obtain

$$
\begin{aligned}
\tau\left(D_{2}\left(S_{n}\right)\right) & =\operatorname{det}(S) \cdot \operatorname{det}\left(P-Q S^{-1} R\right) \\
& =2^{2 n} \operatorname{det}[2 n-n], \\
\tau\left(D_{2}\left(S_{n}\right)\right) & =n \cdot 2^{2 n} .
\end{aligned}
$$

Definition 2. The splitting network $S^{\prime}\left(\Gamma_{1}\right)$ of a network $\Gamma_{1}$ is obtained by taking a vertex set, say $V_{2}$, corresponding to $V\left(\Gamma_{1}\right)$ and then by making all those vertices $u_{i} \in V\left(\Gamma_{1}\right)$ adjacent to the corresponding vertices $v_{i} \in V_{2}$ that preserve the adjacency of $\Gamma_{1}$.
Theorem 3. For all $n$, the complexity of the splitting network $S^{\prime}\left(S_{n}\right)$ is given by

$$
\tau\left(S^{\prime}\left(S_{n}\right)\right)=n \cdot 2^{n-1}
$$

Proof. Let us consider the network $S^{\prime}\left(S_{n}\right)$ with $\left|V\left(S^{\prime}\left(S_{n}\right)\right)\right|=2 n+2$ and $\left|E\left(S^{\prime}\left(S_{n}\right)\right)\right|=3 n$, see the general formation in Figure 3.

Applying Lemma 1, we have

$$
\tau\left(S^{\prime}\left(S_{n}\right)\right)=\frac{1}{(2 n+2)^{2}} \operatorname{det}[(2 n+2) I-\bar{D}+\bar{A}],
$$

where $\bar{D}$ and $\bar{A}$ represent the degree and adjacency matrices of the splitting network $S^{\prime}\left(S_{n}\right)$, respectively. 


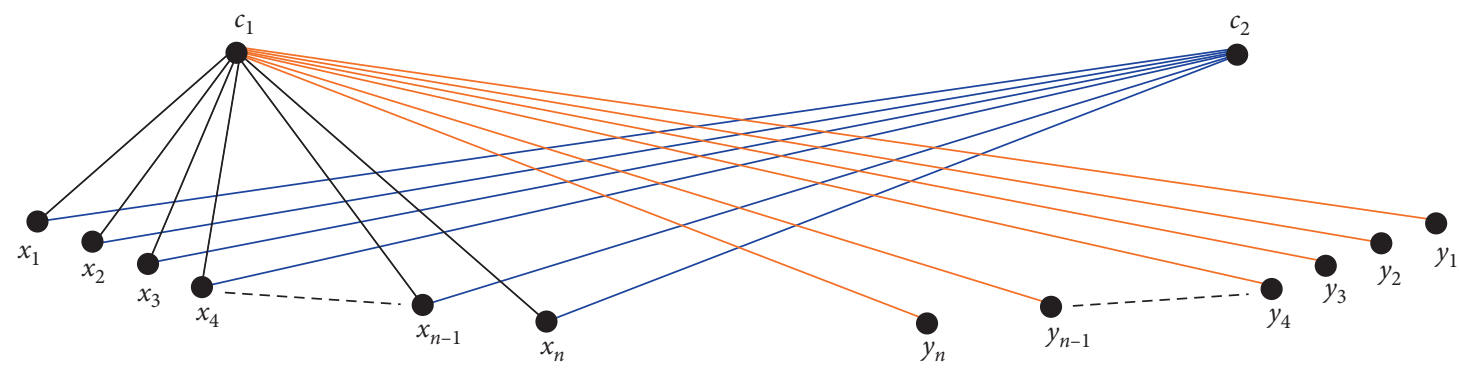

FIgURE 3: The splitting network $S^{\prime}\left(S_{n}\right)$.

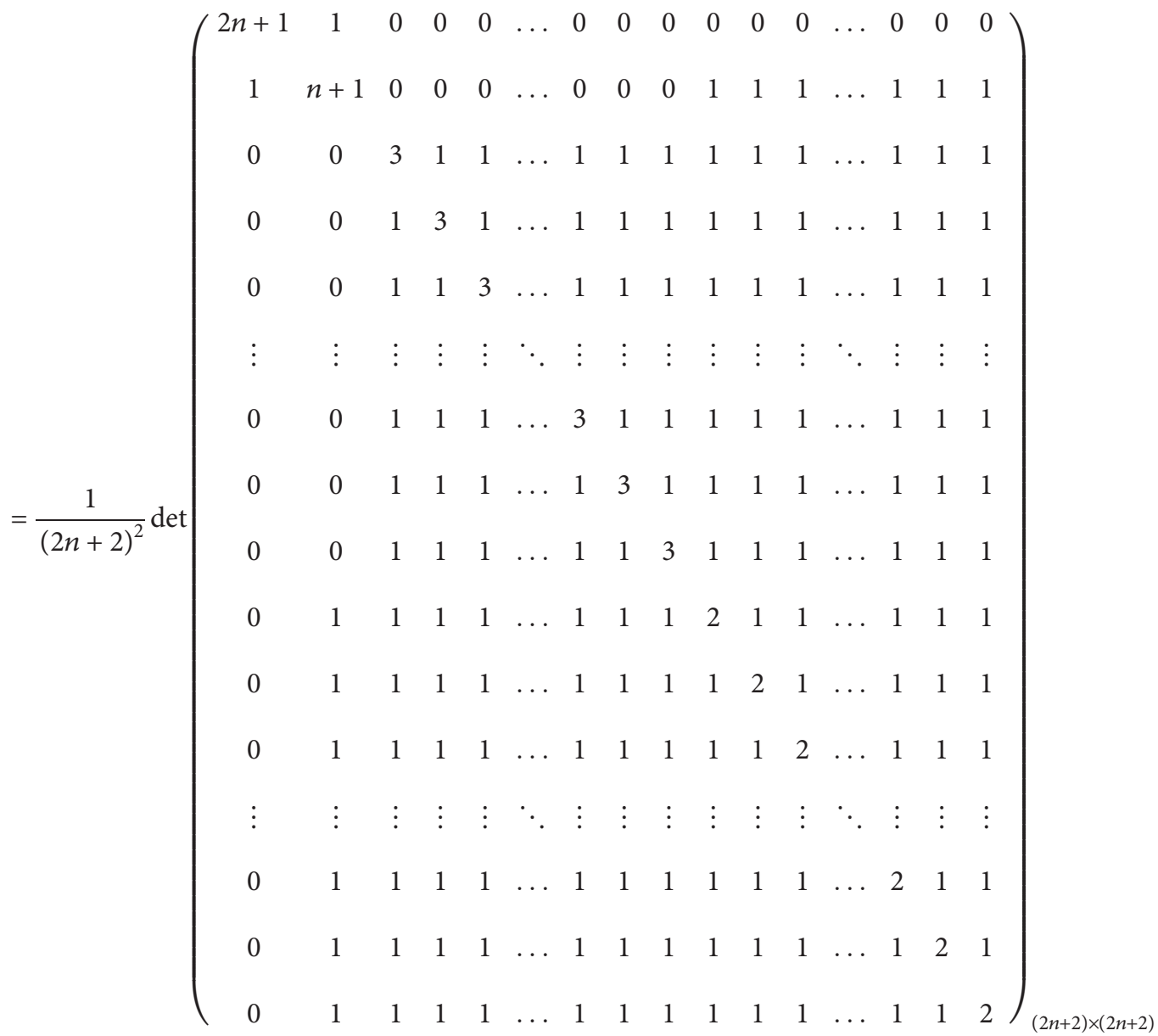




$$
=\operatorname{det}\left(\begin{array}{ccccccccccccccc}
n & -1 & -1 & -1 & \ldots & -1 & -1 & -1 & 0 & 0 & 0 & \ldots & 0 & 0 & 0 \\
-1 & 2 & 0 & 0 & \ldots & 0 & 0 & 0 & 0 & 0 & 0 & \ldots & 0 & 0 & 0 \\
-1 & 0 & 2 & 0 & \ldots & 0 & 0 & 0 & 0 & 0 & 0 & \ldots & 0 & 0 & 0 \\
-1 & 0 & 0 & 2 & \ldots & 0 & 0 & 0 & 0 & 0 & 0 & \ldots & 0 & 0 & 0 \\
\vdots & \vdots & \vdots & \vdots & \ddots & \vdots & \vdots & \vdots & \vdots & \vdots & \vdots & \ddots & \vdots & \vdots & \vdots \\
-1 & 0 & 0 & 0 & \ldots & 2 & 0 & 0 & 0 & 0 & 0 & \ldots & 0 & 0 & 0 \\
-1 & 0 & 0 & 0 & \ldots & 0 & 2 & 0 & 0 & 0 & 0 & \ldots & 0 & 0 & 0 \\
-1 & 0 & 0 & 0 & \ldots & 0 & 0 & 2 & 0 & 0 & 0 & \ldots & 0 & 0 & 0 \\
0 & 0 & 0 & 0 & \ldots & 0 & 0 & 0 & 1 & 0 & 0 & \ldots & 0 & 0 & 0 \\
0 & 0 & 0 & 0 & \ldots & 0 & 0 & 0 & 0 & 1 & 0 & \ldots & 0 & 0 & 0 \\
0 & 0 & 0 & 0 & \ldots & 0 & 0 & 0 & 0 & 0 & 1 & \ldots & 0 & 0 & 0 \\
\vdots & \vdots & \vdots & \vdots & \ddots & \vdots & \vdots & \vdots & \vdots & \vdots & \vdots & \ddots & \vdots & \vdots & \vdots \\
0 & 0 & 0 & 0 & \ldots & 0 & 0 & 0 & 0 & 0 & 0 & \ldots & 1 & 0 & 0 \\
0 & 0 & 0 & 0 & \ldots & 0 & 0 & 0 & 0 & 0 & 0 & \ldots & 0 & 1 & 0 \\
0 & 0 & 0 & 0 & \ldots & 0 & 0 & 0 & 0 & 0 & 0 & \ldots & 0 & 0 & 1
\end{array}\right)_{(2 n+1) \times(2 n+1)}
$$

By using Lemma 4 , we obtain

$$
\begin{aligned}
\tau\left(S^{\prime}\left(S_{n}\right)\right) & =\operatorname{det}(S) \cdot \operatorname{det}\left(P-Q S^{-1} R\right) \\
& =2^{n} \operatorname{det}\left[n-\frac{n}{2}\right] \\
& \Rightarrow \tau\left(S^{\prime}\left(S_{n}\right)\right)=n \cdot 2^{n-1} .
\end{aligned}
$$

Definition 3. The strong product of two networks $\Gamma_{1}=\left(V\left(\Gamma_{1}\right), E\left(\Gamma_{1}\right)\right)$ and $\Gamma_{2}=\left(V\left(\Gamma_{2}\right), E\left(\Gamma_{2}\right)\right)$ is a new network $\Gamma_{1} \otimes \Gamma_{2}$ with vertex and edge sets as follows:

$$
V\left(\Gamma_{1} \otimes \Gamma_{2}\right)=V\left(\Gamma_{1}\right) \times V\left(\Gamma_{2}\right) .
$$

$E\left(\Gamma_{1} \otimes \Gamma_{2}\right)=\left\{\left(u_{1}, u_{2}\right)\left(v_{1}, v_{2}\right)\right.$, whenever $\quad\left(u_{1}=v_{1} \&\right.$ $\left.u_{2} \sim v_{2}\right)$ or $\left(u_{1} \sim v_{1} \& u_{2}=v_{2}\right)$ or $\left(u_{1} \sim v_{1} \& u_{2} \sim v_{2}\right)$, where $\left.\left(u_{1}, u_{2}\right),\left(v_{1}, v_{2}\right) \in V\left(G_{1} \otimes G_{2}\right)\right\}$.
Theorem 4. For all $n$, the complexity of the strong product $S_{n} \otimes P_{2}$ is given by

$$
\tau\left(S_{n} \otimes K_{2}\right)=8^{n}(n+1) .
$$

Proof. Consider the network $S_{n} \otimes K_{2}$ with $\left|V\left(S_{n} \otimes K_{2}\right)\right|=2 n+2$ and $\left|E\left(S_{n} \otimes K_{2}\right)\right|=5 n+1$, see its general formation in Figure 4.

Applying Lemma 1, we have

$$
\tau\left(S_{n} \otimes K_{2}\right)=\frac{1}{(2 n+2)^{2}} \operatorname{det}[(2 n+2) I-\bar{D}+\bar{A}],
$$

where $\bar{D}$ and $\bar{A}$ represent the degree and adjacency matrices of the network $S_{n} \otimes K_{2}$, respectively. 


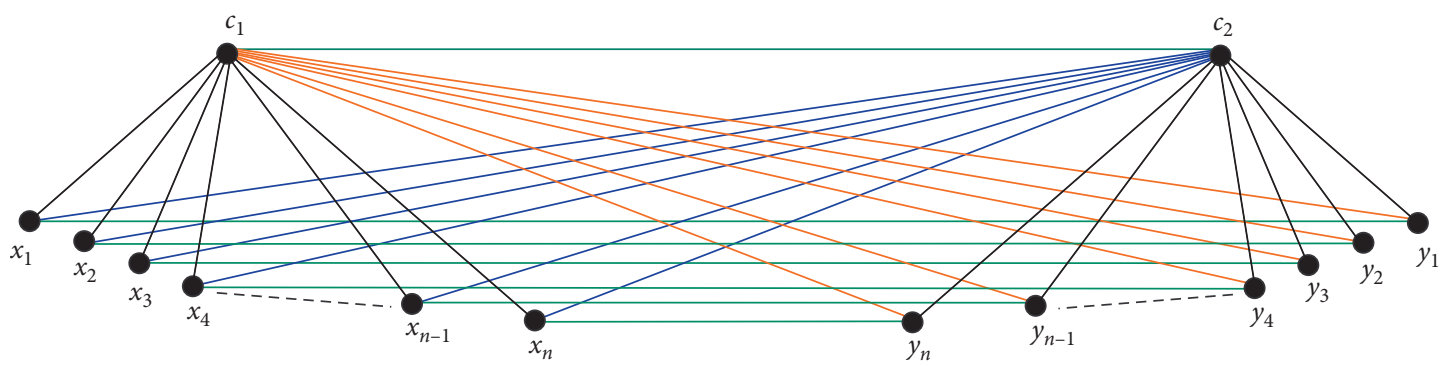

FIGURE 4: The strong product $S_{n} \otimes K_{2}$.

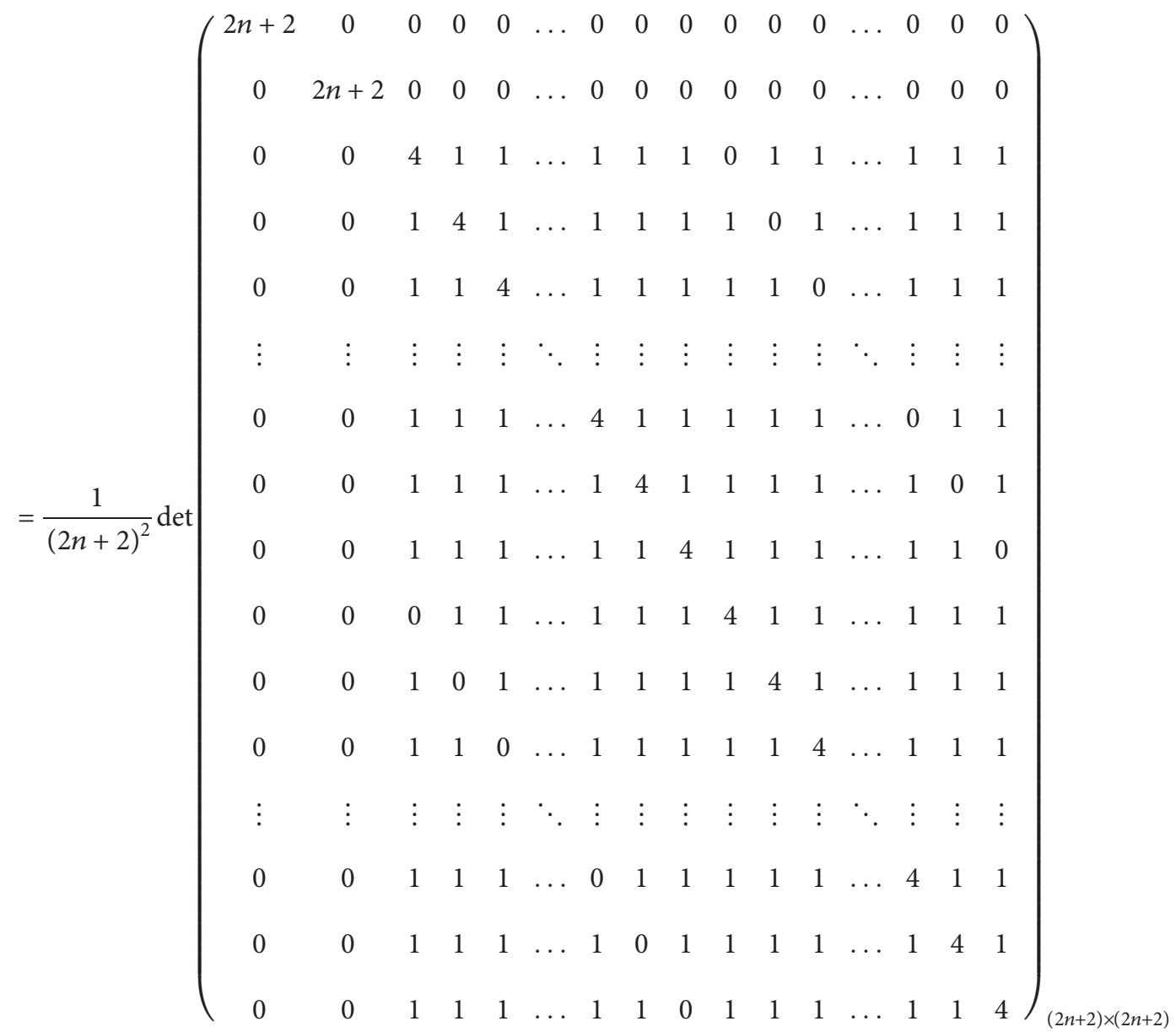




$$
=\operatorname{det}\left(\begin{array}{ccccccccccccccc}
2 n+1 & -1 & -1 & -1 & \ldots & -1 & -1 & -1 & -1 & -1 & -1 & \ldots & -1 & -1 & -1 \\
-1 & 3 & 0 & 0 & \ldots & 0 & 0 & 0 & -1 & 0 & 0 & \ldots & 0 & 0 & 0 \\
-1 & 0 & 3 & 0 & \ldots & 0 & 0 & 0 & 0 & -1 & 0 & \ldots & 0 & 0 & 0 \\
-1 & 0 & 0 & 3 & \ldots & 0 & 0 & 0 & 0 & 0 & -1 & \ldots & 0 & 0 & 0 \\
\vdots & \vdots & \vdots & \vdots & \ddots & \vdots & \vdots & \vdots & \vdots & \vdots & \vdots & \ddots & \vdots & \vdots & \vdots \\
-1 & 0 & 0 & 0 & \ldots & 3 & 0 & 0 & 0 & 0 & 0 & \ldots & -1 & 0 & 0 \\
-1 & 0 & 0 & 0 & \ldots & 0 & 3 & 0 & 0 & 0 & 0 & \ldots & 0 & -1 & 0 \\
-1 & 0 & 0 & 0 & \ldots & 0 & 0 & 3 & 0 & 0 & 0 & \ldots & 0 & 0 & -1 \\
-1 & -1 & 0 & 0 & \ldots & 0 & 0 & 0 & 3 & 0 & 0 & \ldots & 0 & 0 & 0 \\
-1 & 0 & -1 & 0 & \ldots & 0 & 0 & 0 & 0 & 3 & 0 & \ldots & 0 & 0 & 0 \\
-1 & 0 & 0 & -1 & \ldots & 0 & 0 & 0 & 0 & 0 & 3 & \ldots & 0 & 0 & 0 \\
\vdots & \vdots & \vdots & \vdots & \ddots & \vdots & \vdots & \vdots & \vdots & \vdots & \vdots & \ddots & \vdots & \vdots & \vdots \\
-1 & 0 & 0 & 0 & \ldots & -1 & 0 & 0 & 0 & 0 & 0 & \ldots & 3 & 0 & 0 \\
-1 & 0 & 0 & 0 & \ldots & 0 & -1 & 0 & 0 & 0 & 0 & \ldots & 0 & 3 & 0 \\
-1 & 0 & 0 & 0 & \ldots & 0 & 0 & -1 & 0 & 0 & 0 & \ldots & 0 & 0 & 3
\end{array}\right)_{(2 n+1) \times(2 n+1)}
$$

By using Lemma 4, we obtain

$$
\begin{aligned}
& \tau\left(S_{n} \otimes K_{2}\right)=\operatorname{det}(S) \cdot \operatorname{det}\left(P-Q S^{-1} R\right) \\
& =3^{n} \cdot\left(\frac{-4}{3}\right)^{n+1} \operatorname{det}\left(\begin{array}{ccccccccc}
\frac{5 n+3}{-4} & 1 & 1 & 1 & \ldots & 1 & 1 & 1 & 1 \\
1 & -2 & 0 & 0 & \ldots & 0 & 0 & 0 & 0 \\
1 & 0 & -2 & 0 & \ldots & 0 & 0 & 0 & 0 \\
1 & 0 & 0 & -2 & \ldots & 0 & 0 & 0 & 0 \\
\vdots & \vdots & \vdots & \vdots & \ddots & \vdots & \vdots & \vdots & \vdots \\
1 & 0 & 0 & 0 & \ldots & -2 & 0 & 0 & 0 \\
1 & 0 & 0 & 0 & \ldots & 0 & -2 & 0 & 0 \\
1 & 0 & 0 & 0 & \ldots & 0 & 0 & -2 & 0 \\
1 & 0 & 0 & 0 & \ldots & 0 & 0 & 0 & -2
\end{array}\right)_{(n+1) \times(n+1)} \\
& =3^{n} \cdot\left(\frac{-4}{3}\right)^{n+1} \operatorname{det}(A)_{(n+1) \times(n+1)} \text {. }
\end{aligned}
$$


Calculating the values of $\operatorname{det}(A)$ for $n=1,2,3, \ldots, 10$. We obtain $\operatorname{det}(A)=3,-9,24,-60,144,-336,768,-1728,3840$, -8448 . Upon generalizing for $n$, we obtain $\operatorname{det}(A)=$ $\left(3(-2)^{n}(n+1) /-4\right)$.

$$
\Rightarrow \tau\left(S_{n} \otimes K_{2}\right)=3^{n} \cdot\left(\frac{-4}{3}\right)^{n+1}\left(\frac{3(-2)^{n}(n+1)}{-4}\right) .
$$

Finally, we obtain

$$
\tau\left(S_{n} \otimes K_{2}\right)=8^{n}(n+1) .
$$

We define here a new operation namely vortex $V^{\prime}(\Gamma)$ of a network $\Gamma$.

Definition 4. The vortex $V^{\prime}\left(G_{1}\right)$ of a network $\Gamma_{1}$ is obtained by taking a vertex set, say $V_{2}$, corresponding to $V\left(\Gamma_{1}\right)$ and then by making all those vertices $u_{i} \in V\left(\Gamma_{1}\right)$ adjacent to those vertices $v_{i} \in V_{2}$ that do not preserve the adjacency of $\Gamma_{1}$.

Theorem 5. For all $n$, the complexity of the vortex $V^{\prime}\left(S_{n}\right)$ is given by

$$
\tau\left(V^{\prime}\left(S_{n}\right)\right)=n^{n}(n+1)^{n-1} .
$$

Proof. Consider the network $V^{\prime}\left(S_{n}\right)$ with $\left|V\left(V^{\prime}\left(S_{n}\right)\right)\right|=$ $2 n+2$ and $\left|E\left(V^{\prime}\left(S_{n}\right)\right)\right|=n^{2}+n+1$, see Figure 5 . Applying Lemma 1, we have

$$
\tau\left(V^{\prime}\left(S_{n}\right)\right)=\frac{1}{(2 n+2)^{2}} \operatorname{det}[(2 n+2) I-\bar{D}+\bar{A}],
$$

where $\bar{D}$ and $\bar{A}$ represent the degree and adjacency matrices of the vortex $V^{\prime}\left(S_{n}\right)$, respectively.

$$
=\frac{1}{(2 n+2)^{2}} \operatorname{det}\left(\begin{array}{cccccccccccccccc}
n+2 & 0 & 0 & 0 & 0 & \ldots & 0 & 0 & 0 & 1 & 1 & 1 & \ldots & 1 & 1 & 1 \\
0 & 2 & 1 & 1 & 1 & \ldots & 1 & 1 & 1 & 1 & 1 & 1 & \ldots & 1 & 1 & 1 \\
0 & 1 & n+2 & 1 & 1 & \ldots & 1 & 1 & 1 & 0 & 0 & 0 & \ldots & 0 & 0 & 0 \\
0 & 1 & 1 & n+2 & 1 & \ldots & 1 & 1 & 1 & 0 & 0 & 0 & \ldots & 0 & 0 & 0 \\
0 & 1 & 1 & 1 & n+2 & \ldots & 1 & 1 & 1 & 0 & 0 & 0 & \ldots & 0 & 0 & 0 \\
\vdots & \vdots & \vdots & \vdots & \vdots & \ddots & \vdots & \vdots & \vdots & \vdots & \vdots & \vdots & \ddots & \vdots & \vdots & \vdots \\
0 & 1 & 1 & 1 & 1 & \ldots & n+2 & 1 & 1 & 0 & 0 & 0 & \ldots & 0 & 0 & 0 \\
0 & 1 & 1 & 1 & 1 & \ldots & 1 & n+2 & 1 & 0 & 0 & 0 & \ldots & 0 & 0 & 0 \\
1 & 1 & 0 & 0 & 0 & \ldots & 0 & 0 & 0 & n+1 & 1 & 1 & \ldots & 1 & 1 & 1 \\
1 & 1 & 0 & 0 & 0 & \ldots & 0 & 0 & 0 & 1 & n+1 & 1 & \ldots & 1 & 1 & 1 \\
1 & 1 & 0 & 0 & 0 & \ldots & 0 & 0 & 0 & 1 & 1 & n+1 & \ldots & 1 & 1 & 1 \\
\vdots & \vdots & \vdots & \vdots & \vdots & \ddots & \vdots & \vdots & \vdots & \vdots & \vdots & \vdots & \ddots & \vdots & \vdots & \vdots \\
1 & 1 & 0 & 0 & 0 & \ldots & 0 & 0 & 0 & 1 & 1 & 1 & \ldots & n+1 & 1 & 1 \\
1 & 1 & 0 & 0 & 0 & \ldots & 0 & 0 & 0 & 1 & 1 & 1 & \ldots & 1 & n+1 & 1 \\
1 & 1 & 0 & 0 & 0 & \ldots & 0 & 0 & 0 & 1 & 1 & 1 & \ldots & 1 & 1 & n+1
\end{array}\right)
$$




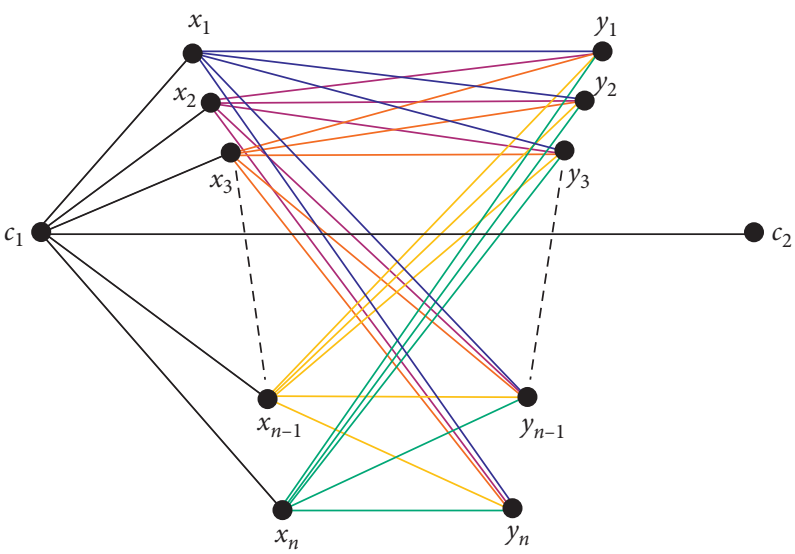

Figure 5: The vortex network $V^{\prime}\left(S_{n}\right)$.

$$
\begin{aligned}
& =\operatorname{det}\left(\begin{array}{ccccccccccccccc}
1 & 0 & 0 & 0 & \ldots & 0 & 0 & 0 & 0 & 0 & 0 & \ldots & 0 & 0 & 0 \\
0 & n+1 & 0 & 0 & \ldots & 0 & 0 & 0 & -1 & -1 & -1 & \ldots & -1 & -1 & -1 \\
0 & 0 & n+1 & 0 & \ldots & 0 & 0 & 0 & -1 & -1 & -1 & \ldots & -1 & -1 & -1 \\
0 & 0 & 0 & n+1 & \ldots & 0 & 0 & 0 & -1 & -1 & -1 & \ldots & -1 & -1 & -1 \\
\vdots & \vdots & \vdots & \vdots & \ddots & \vdots & \vdots & \vdots & \vdots & \vdots & \vdots & \ddots & \vdots & \vdots & \vdots \\
0 & 0 & 0 & 0 & \ldots & n+1 & 0 & 0 & -1 & -1 & -1 & \ldots & -1 & -1 & -1 \\
0 & 0 & 0 & 0 & \ldots & 0 & n+1 & 0 & -1 & -1 & -1 & \ldots & -1 & -1 & -1 \\
0 & 0 & 0 & 0 & \ldots & 0 & 0 & n+1 & -1 & -1 & -1 & \ldots & -1 & -1 & -1 \\
0 & -1 & -1 & -1 & \ldots & -1 & -1 & -1 & n & 0 & 0 & \ldots & 0 & 0 & 0 \\
0 & -1 & -1 & -1 & \ldots & -1 & -1 & -1 & 0 & n & 0 & \ldots & 0 & 0 & 0 \\
0 & -1 & -1 & -1 & \ldots & -1 & -1 & -1 & 0 & 0 & n & \ldots & 0 & 0 & 0 \\
\vdots & \vdots & \vdots & \vdots & \ddots & \vdots & \vdots & \vdots & \vdots & \vdots & \vdots & \ddots & \vdots & \vdots & \vdots \\
0 & -1 & -1 & -1 & \ldots & -1 & -1 & -1 & 0 & 0 & 0 & \ldots & n & 0 & 0 \\
0 & -1 & -1 & -1 & \ldots & -1 & -1 & -1 & 0 & 0 & 0 & \ldots & 0 & n & 0 \\
0 & -1 & -1 & -1 & \ldots & -1 & -1 & -1 & 0 & 0 & 0 & \ldots & 0 & 0 & n
\end{array}\right)_{(2 n+1) \times(2 n+1)} \\
& \Rightarrow \tau\left(V^{\prime}\left(S_{n}\right)\right)=\operatorname{det}\left(\begin{array}{cc}
P_{(n+1) \times(n+1)} & Q_{(n+1) \times n} \\
R_{n \times(n+1)} & S_{n \times n}
\end{array}\right)_{(2 n+1) \times(2 n+1)} .
\end{aligned}
$$

By using Lemma 4, we obtain 


$$
\begin{aligned}
& \tau\left(V^{\prime}\left(S_{n}\right)\right)=\operatorname{det}(S) \cdot \operatorname{det}\left(P-Q S^{-1} R\right) \\
& =n^{n} \operatorname{det}\left(\begin{array}{ccccccccc}
1 & 0 & 0 & 0 & \ldots & 0 & 0 & 0 & 0 \\
0 & n & -1 & -1 & \ldots & -1 & -1 & -1 & -1 \\
0 & -1 & n & -1 & \ldots & -1 & -1 & -1 & -1 \\
0 & -1 & -1 & n & \ldots & -1 & -1 & -1 & -1 \\
\vdots & \vdots & \vdots & \vdots & \ddots & \vdots & \vdots & \vdots & \vdots \\
0 & -1 & -1 & -1 & \ldots & n & -1 & -1 & -1 \\
0 & -1 & -1 & -1 & \ldots & -1 & n & -1 & -1 \\
0 & -1 & -1 & -1 & \ldots & -1 & -1 & n & -1 \\
0 & -1 & -1 & -1 & \ldots & -1 & -1 & -1 & n
\end{array}\right)_{(n+1) \times(n+1)} \\
& =n^{n}(-1)^{n} \operatorname{det}\left(\begin{array}{cccccccc}
-n & 1 & 1 & \ldots & 1 & 1 & 1 & 1 \\
1 & -n & 1 & \ldots & 1 & 1 & 1 & 1 \\
1 & 1 & -n & \ldots & 1 & 1 & 1 & 1 \\
\vdots & \vdots & \vdots & \ddots & \vdots & \vdots & \vdots & \vdots \\
1 & 1 & 1 & \ldots & -n & 1 & 1 & 1 \\
1 & 1 & 1 & \ldots & 1 & -n & 1 & 1 \\
1 & 1 & 1 & \ldots & 1 & 1 & -n & 1 \\
1 & 1 & 1 & \ldots & 1 & 1 & 1 & -n
\end{array}\right)_{n \times n} \\
& \tau\left(S_{n}+K_{2}\right)=3^{n-2}\left[(2 n+6)^{2}-(n+3)^{2}\right] .
\end{aligned}
$$

Applying Lemma 3,

$$
\begin{aligned}
& =n^{n}(-1)^{n}(-n+n-1)(-n-1)^{n-1} \\
& \Rightarrow \tau\left(V^{\prime}\left(S_{n}\right)\right)=n^{n}(n+1)^{n-1} .
\end{aligned}
$$

Theorem 6. For all $n$, the complexity of the network $S_{n}+K_{2}$ is given by

Proof. Consider the network $S_{n}+K_{2}$ with $\left|V\left(S_{n}+K_{2}\right)\right|=$ $n+3$ and $\left|E\left(S_{n}+K_{2}\right)\right|=3(n+1)$, see Figure 6 . Applying Lemma 1 , we have 


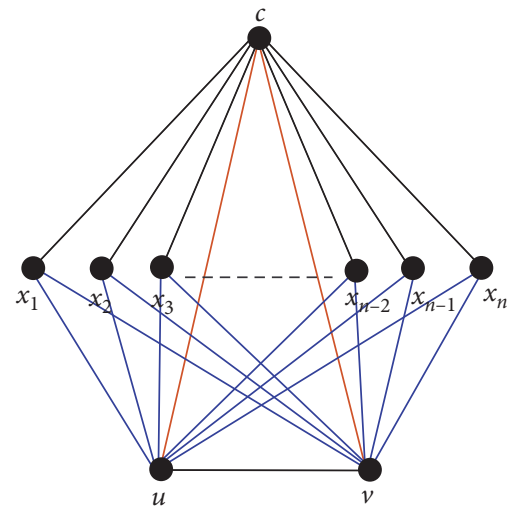

FIgURE 6: The network $S_{n}+K_{2}$.

$$
\begin{aligned}
\tau\left(S_{n}+K_{2}\right) & =\frac{1}{(n+3)^{2}} \operatorname{det}[(n+3) I-\bar{D}+\bar{A}] \\
& =\frac{1}{(n+3)^{2}} \operatorname{det}\left(\begin{array}{cccccccccccccc}
n+3 & 0 & 0 & 0 & 0 & 0 & 0 & \ldots & 0 & 0 & 0 & 0 \\
0 & n+3 & 0 & 0 & 0 & 0 & 0 & \ldots & 0 & 0 & 0 & 0 \\
0 & 0 & n+3 & 0 & 0 & 0 & 0 & \ldots & 0 & 0 & 0 & 0 \\
0 & 0 & 0 & 4 & 1 & 1 & 1 & \ldots & 1 & 1 & 1 & 1 \\
0 & 0 & 0 & 1 & 4 & 1 & 1 & \ldots & 1 & 1 & 1 & 1 \\
0 & 0 & 0 & 1 & 1 & 4 & 1 & \ldots & 1 & 1 & 1 & 1 \\
\vdots & \vdots & 0 & 1 & 1 & 1 & 4 & \ldots & 1 & 1 & 1 & 1 \\
0 & 0 & 0 & 1 & 1 & 1 & 1 & \ldots & 4 & 1 & 1 & 1 \\
0 & 0 & 0 & 1 & 1 & 1 & 6 & \ldots & 1 & 4 & 1 & 1 \\
0 & 0 & 0 & 0 & 1 & 1 & 1 & 6 & \ldots & 1 & 1 & 1 & 4
\end{array}\right)_{(n+3) \times(n+3)}
\end{aligned}
$$




$$
\begin{gathered}
=\operatorname{det}\left(\begin{array}{ccccccccccc}
n+2 & -1 & -1 & -1 & -1 & -1 & \ldots & -1 & -1 & -1 & -1 \\
-1 & n+2 & -1 & -1 & -1 & -1 & \ldots & -1 & -1 & -1 & -1 \\
-1 & -1 & 3 & 0 & 0 & 0 & \ldots & 0 & 0 & 0 & 0 \\
-1 & -1 & 0 & 3 & 0 & 0 & \ldots & 0 & 0 & 0 & 0 \\
-1 & -1 & 0 & 0 & 3 & 0 & \ldots & 0 & 0 & 0 & 0 \\
-1 & -1 & 0 & 0 & 0 & 3 & \ldots & 0 & 0 & 0 & 0 \\
\vdots & \vdots & \vdots & \vdots & \vdots & \vdots & \vdots & \ddots & \vdots & \vdots & \vdots \\
-1 & -1 & 0 & 0 & 0 & 0 & \ldots & 3 & 0 & 0 & 0 \\
-1 & -1 & 0 & 0 & 0 & 0 & \ldots & 0 & 3 & 0 & 0 \\
-1 & -1 & 0 & 0 & 0 & 0 & \ldots & 0 & 0 & 3 & 0 \\
-1 & -1 & 5 & 0 & 0 & 0 & \ldots & 0 & 0 & 0 & 3
\end{array}\right)_{(n+2) \times(n+2)} \\
\Rightarrow \tau\left(S_{n}+K_{2}\right)=\operatorname{det}\left(\begin{array}{cccccc}
P_{2 \times 2} & Q_{2 \times n} \\
R_{n \times 2} & S_{n \times n}
\end{array}\right)_{(n+2) \times(n+2)} \\
\end{gathered}
$$

By using Lemma 4 , we obtain

$$
\begin{array}{r}
\tau\left(S_{n}+K_{2}\right)=\operatorname{det}(S) \cdot \operatorname{det}\left(P-Q S^{-1} R\right) \\
=3^{n} \operatorname{det}\left(\begin{array}{cc}
\frac{2 n+6}{3} & -\left(\frac{n+3}{3}\right) \\
-\left(\frac{n+3}{3}\right) & \frac{2 n+6}{3}
\end{array}\right) \\
\Rightarrow \tau\left(S_{n}+K_{2}\right)=3^{n-2}\left[(2 n+6)^{2}-(n+3)^{2}\right] .
\end{array}
$$

Theorem 7. For $n \geq 3$, the complexity of an edge-subdivision of the wheel $W_{n}^{\prime}$ is given by

$$
\tau\left(W_{n}^{\prime}\right)=2^{2 n}-2^{n+1}+1 .
$$

(41) Proof. Consider the edge-subdivision of wheel $W_{n}^{\prime}$ with $\left|V\left(W_{n}^{\prime}\right)\right|=2 n+1,\left|E\left(W_{n}^{\prime}\right)\right|=3 n$, see Figure 7.

Applying Lemma 1 , we have

$$
\tau\left(W_{n}^{\prime}\right)=\frac{1}{(2 n+1)^{2}} \operatorname{det}[(2 n+1) I-\bar{D}+\bar{A}],
$$




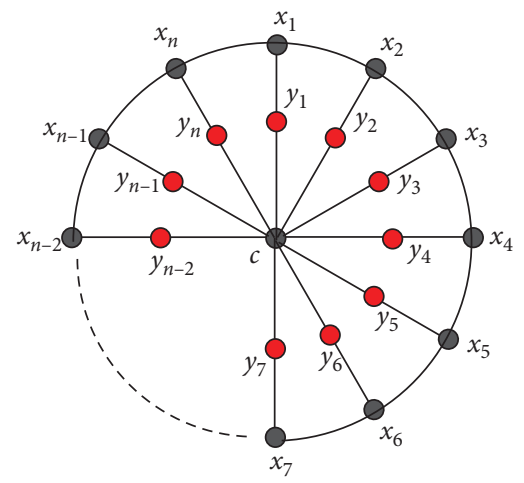

Figure 7: An edge-subdivision of the network $W_{n}$.

where $\bar{D}$ and $\bar{A}$ represent the degree and adjacency matrices of the network $W_{n}^{\prime}$, respectively.

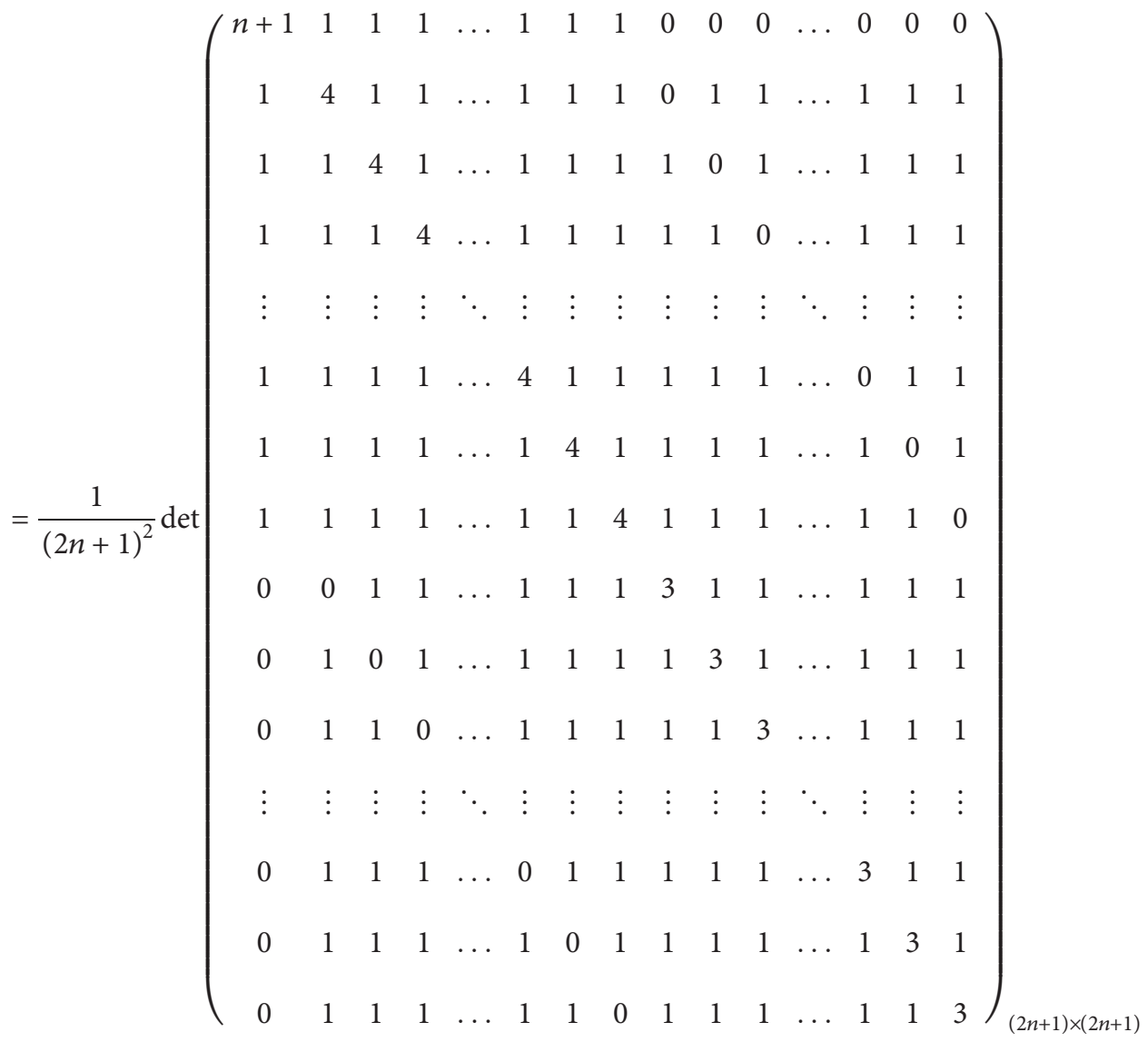




$$
=\operatorname{det}\left(\begin{array}{cccccccccccccc}
3 & -1 & 0 & \ldots & 0 & 0 & -1 & -1 & 0 & 0 & \ldots & 0 & 0 & 0 \\
-1 & 3 & -1 & \ldots & 0 & 0 & 0 & 0 & -1 & 0 & \ldots & 0 & 0 & 0 \\
0 & -1 & 3 & \ldots & 0 & 0 & 0 & 0 & 0 & -1 & \ldots & 0 & 0 & 0 \\
\vdots & \vdots & \vdots & \ddots & \vdots & \vdots & \vdots & \vdots & \vdots & \vdots & \ddots & \vdots & \vdots & \vdots \\
0 & 0 & 0 & \ldots & 3 & -1 & 0 & 0 & 0 & 0 & \ldots & -1 & 0 & 0 \\
0 & 0 & 0 & \ldots & -1 & 3 & -1 & 0 & 0 & 0 & \ldots & 0 & -1 & 0 \\
-1 & 0 & 0 & \ldots & 0 & -1 & 3 & 0 & 0 & 0 & \ldots & 0 & 0 & -1 \\
-1 & 0 & 0 & \ldots & 0 & 0 & 0 & 2 & 0 & 0 & \ldots & 0 & 0 & 0 \\
0 & -1 & 0 & \ldots & 0 & 0 & 0 & 0 & 2 & 0 & \ldots & 0 & 0 & 0 \\
0 & 0 & -1 & \ldots & 0 & 0 & 0 & 0 & 0 & 2 & \ldots & 0 & 0 & 0 \\
\vdots & \vdots & \vdots & \ddots & \vdots & \vdots & \vdots & \vdots & \vdots & \vdots & \ddots & \vdots & \vdots & \vdots \\
0 & 0 & 0 & \ldots & -1 & 0 & 0 & 0 & 0 & 0 & \ldots & 2 & 0 & 0 \\
0 & 0 & 0 & \ldots & 0 & -1 & 0 & 0 & 0 & 0 & \ldots & 0 & 2 & 0 \\
0 & 0 & 0 & \ldots & 0 & 0 & -1 & 0 & 0 & 0 & \ldots & 0 & 0 & 2
\end{array}\right)_{2 n \times 2 n}
$$

By using Lemma 4, we obtain $\tau\left(W_{n}^{\prime}\right)=\operatorname{det}(S) \cdot \operatorname{det}\left(P-Q S^{-1} R\right)$

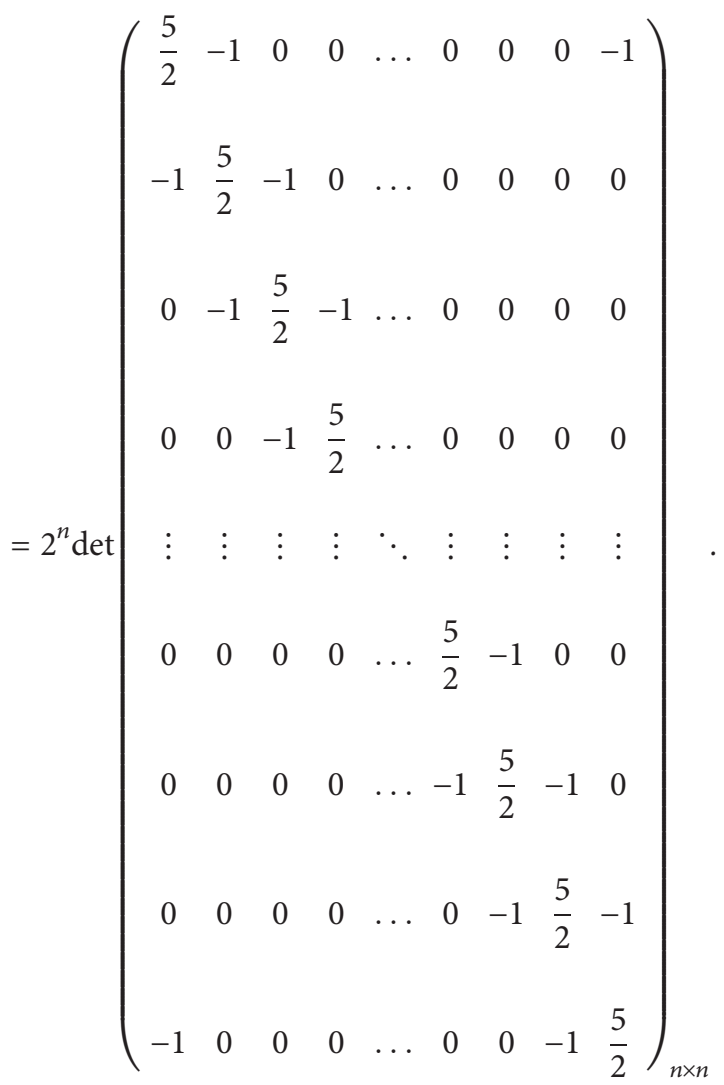

Using Lemma 2,

$$
\begin{aligned}
& =2^{n} \cdot 2\left[T_{n}\left(\frac{5}{4}\right)-1\right] \\
& =2^{n}\left[2^{n}+\frac{1}{2^{n}}-2\right] \\
& \Rightarrow \tau\left(W_{n}^{\prime}\right)=2^{2 n}-2^{n+1}+1 .
\end{aligned}
$$

Theorem 8. For $n \geq 3$, the complexity of the rooted product. $W_{n} \circ K_{2}$. is given as

$$
\tau\left(W_{n} \circ K_{2}\right)=\left(\frac{3+\sqrt{5}}{2}\right)^{n}+\left(\frac{3-\sqrt{5}}{2}\right)^{n}-2 .
$$

Proof. Consider the network $W_{n} \circ K_{2}$ with $\left|V\left(W_{n} \circ K_{2}\right)\right|=$ $2 n+1$ and $\left|E\left(W_{n} \circ K_{2}\right)\right|=3 n$. The general formation is shown in Figure 8.

Applying Lemma 1, we have

$$
\tau\left(W_{n} \circ K_{2}\right)=\frac{1}{(2 n+1)^{2}} \operatorname{det}[(2 n+1) I-\bar{D}+\bar{A}],
$$

where $\bar{D}$ and $\bar{A}$ represent the degree and adjacency matrices of the network $W_{n} \circ K_{2}$, respectively. 


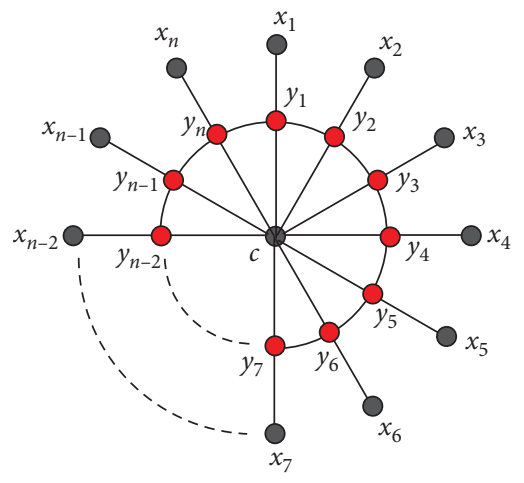

Figure 8: The general formation of the rooted product $W_{n} \circ K_{2}$.

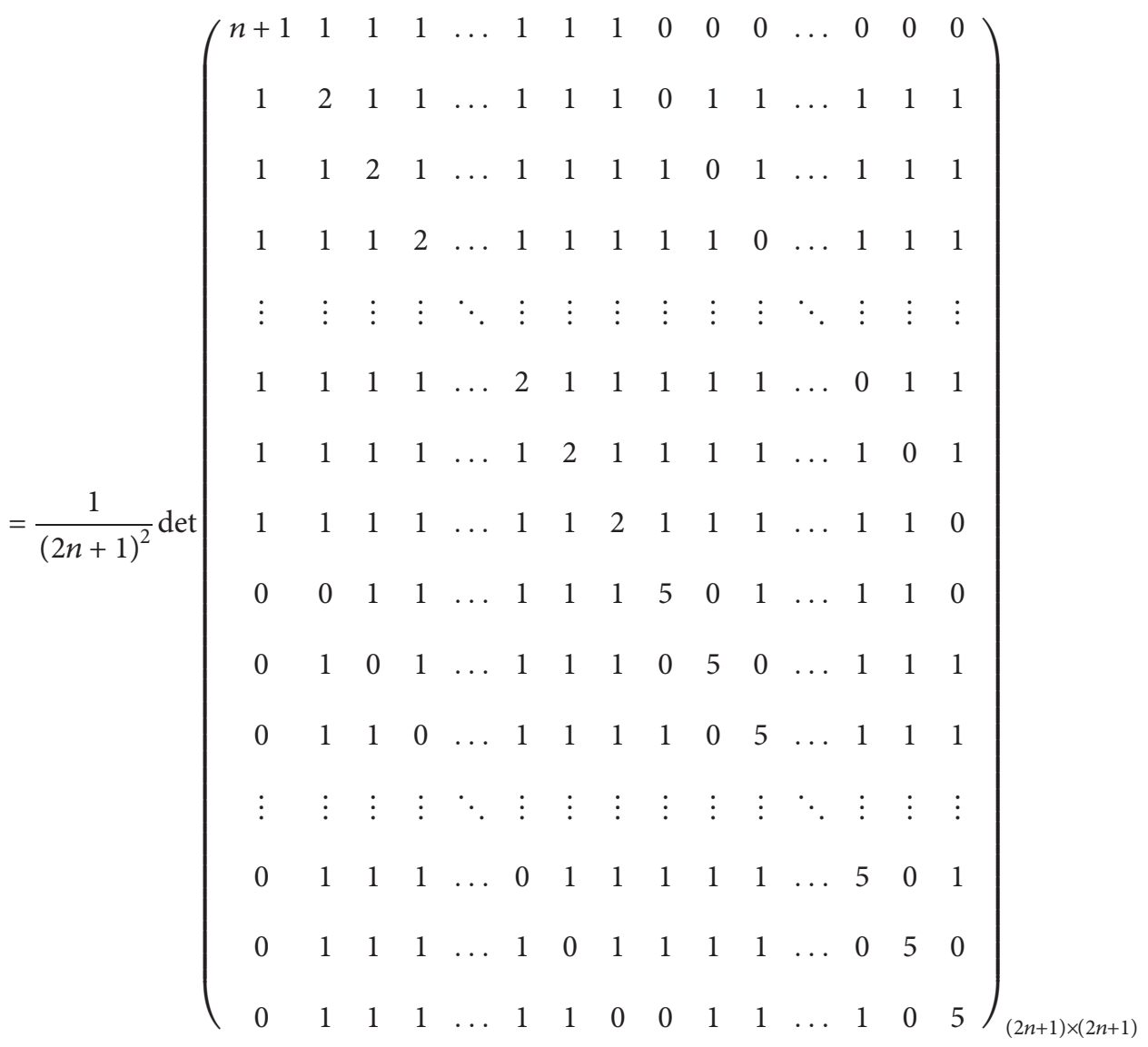




$$
=\operatorname{det}\left(\begin{array}{cccccccccccccc}
1 & 0 & 0 & \ldots & 0 & 0 & 0 & -1 & 0 & 0 & \ldots & 0 & 0 & 0 \\
0 & 1 & 0 & \ldots & 0 & 0 & 0 & 0 & -1 & 0 & \ldots & 0 & 0 & 0 \\
0 & 0 & 1 & \ldots & 0 & 0 & 0 & 0 & 0 & -1 & \ldots & 0 & 0 & 0 \\
\vdots & \vdots & \vdots & \ddots & \vdots & \vdots & \vdots & \vdots & \vdots & \vdots & \ddots & \vdots & \vdots & \vdots \\
0 & 0 & 0 & \ldots & 1 & 0 & 0 & 0 & 0 & 0 & \ldots & -1 & 0 & 0 \\
0 & 0 & 0 & \ldots & 0 & 1 & 0 & 0 & 0 & 0 & \ldots & 0 & -1 & 0 \\
0 & 0 & 0 & \ldots & 0 & 0 & 1 & 0 & 0 & 0 & \ldots & 0 & 0 & -1 \\
-1 & 0 & 0 & \ldots & 0 & 0 & 0 & 4 & -1 & 0 & \ldots & 0 & 0 & -1 \\
0 & -1 & 0 & \ldots & 0 & 0 & 0 & -1 & 4 & -1 & \ldots & 0 & 0 & 0 \\
0 & 0 & -1 & \ldots & 0 & 0 & 0 & 0 & -1 & 4 & \ldots & 0 & 0 & 0 \\
\vdots & \vdots & \vdots & \ddots & \vdots & \vdots & \vdots & \vdots & \vdots & \vdots & \ddots & \vdots & \vdots & \vdots \\
0 & 0 & 0 & \ldots & -1 & 0 & 0 & 0 & 0 & 0 & \ldots & 4 & -1 & 0 \\
0 & 0 & 0 & \ldots & 0 & -1 & 0 & 0 & 0 & 0 & \ldots & -1 & 4 & -1 \\
0 & 0 & 0 & \ldots & 0 & 0 & -1 & -1 & 0 & 0 & \ldots & 0 & -1 & 4
\end{array}\right)_{2 n \times 2 n}
$$

By using Lemma 4, we obtain $\tau\left(W_{n} \circ K_{2}\right)=\operatorname{det}(P) \cdot \operatorname{det}\left(S-R P^{-1} Q\right)$

$$
=1 \cdot \operatorname{det}\left(\begin{array}{ccccccccc}
3 & -1 & 0 & 0 & \ldots & 0 & 0 & 0 & -1 \\
-1 & 3 & -1 & 0 & \ldots & 0 & 0 & 0 & 0 \\
0 & -1 & 3 & -1 & \ldots & 0 & 0 & 0 & 0 \\
0 & 0 & -1 & 3 & \ldots & 0 & 0 & 0 & 0 \\
\vdots & \vdots & \vdots & \vdots & \ddots & \vdots & \vdots & \vdots & \vdots \\
0 & 0 & 0 & 0 & \ldots & 3 & -1 & 0 & 0 \\
0 & 0 & 0 & 0 & \ldots & -1 & 3 & -1 & 0 \\
0 & 0 & 0 & 0 & \ldots & 0 & -1 & 3 & -1 \\
-1 & 0 & 0 & 0 & \ldots & 0 & 0 & -1 & 3
\end{array}\right)_{n \times n}
$$

Using Lemma 2,

$$
\begin{aligned}
& =2\left[T_{n}\left(\frac{3}{2}\right)-1\right] \\
& \Rightarrow \tau\left(W_{n} \circ K_{2}\right)=\left(\frac{3+\sqrt{5}}{2}\right)^{n}+\left(\frac{3-\sqrt{5}}{2}\right)^{n}-2 .
\end{aligned}
$$

Definition 5. The vertex semitotal network $R(\Gamma)$ of a network $\Gamma$ is obtained by taking a vertex corresponding to each edge of $\Gamma$ and then by joining these new vertices to the end points of their corresponding edges.

Theorem 9. For all $n$, the complexity of the vertex semitotal network $R\left(S_{n}\right)$ is given as

$$
\tau\left(R\left(S_{n}\right)\right)=3^{n}
$$

Proof. Consider the vertex semitotal network $R\left(S_{n}\right)$ with $\left|V\left(R\left(S_{n}\right)\right)\right|=2 n+1$ and $\left|E\left(R\left(S_{n}\right)\right)\right|=3 n$, see Figure 9 for its general formation.

Applying Lemma 1, we have

$$
\tau\left(R\left(S_{n}\right)\right)=\frac{1}{(2 n+1)^{2}} \operatorname{det}[(2 n+1) I-\bar{D}+\bar{A}],
$$

where $\bar{D}$ and $\bar{A}$ represent the degree and adjacency matrices of $R\left(S_{n}\right)$, respectively. 


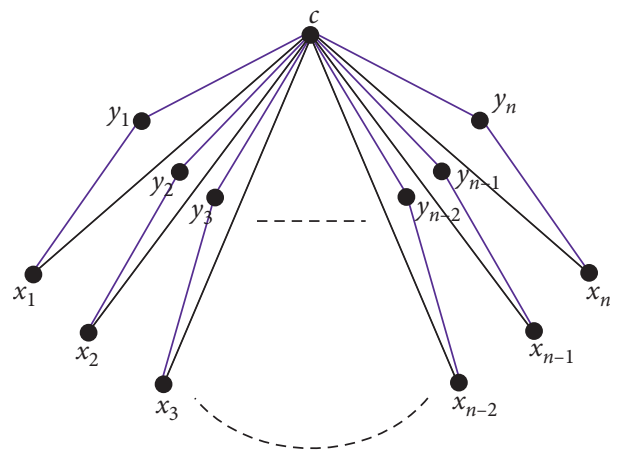

FIgURE 9: The vertex-semitotal network $R\left(S_{n}\right)$.

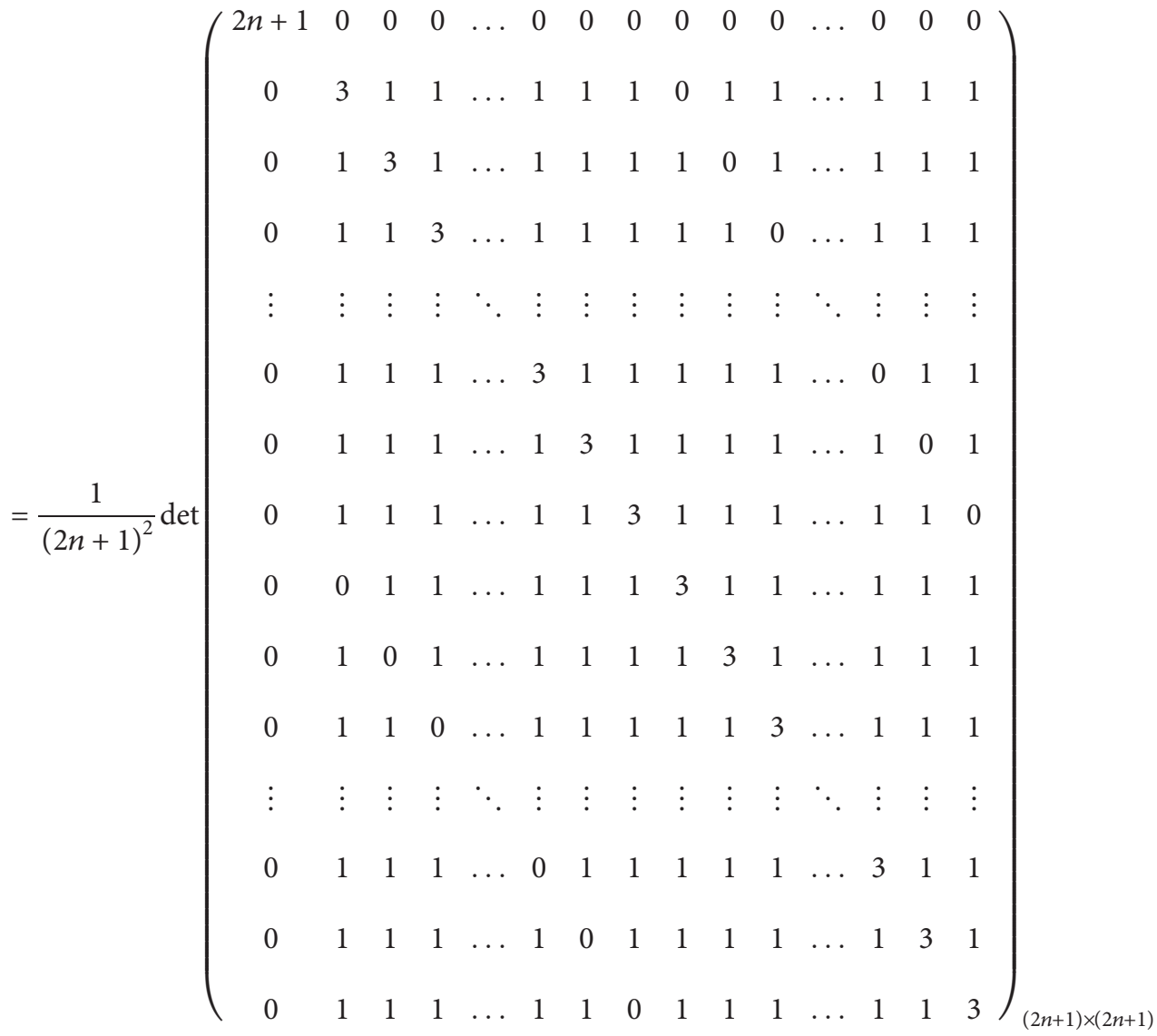




$$
=\operatorname{det}\left(\begin{array}{cccccccccccccc}
2 & 0 & 0 & \ldots & 0 & 0 & 0 & -1 & 0 & 0 & \ldots & 0 & 0 & 0 \\
0 & 2 & 0 & \ldots & 0 & 0 & 0 & 0 & -1 & 0 & \ldots & 0 & 0 & 0 \\
0 & 0 & 2 & \ldots & 0 & 0 & 0 & 0 & 0 & -1 & \ldots & 0 & 0 & 0 \\
\vdots & \vdots & \vdots & \ddots & \vdots & \vdots & \vdots & \vdots & \vdots & \vdots & \ddots & \vdots & \vdots & \vdots \\
0 & 0 & 0 & \ldots & 2 & 0 & 0 & 0 & 0 & 0 & \ldots & -1 & 0 & 0 \\
0 & 0 & 0 & \ldots & 0 & 2 & 0 & 0 & 0 & 0 & \ldots & 0 & -1 & 0 \\
0 & 0 & 0 & \ldots & 0 & 0 & 2 & 0 & 0 & 0 & \ldots & 0 & 0 & -1 \\
-1 & 0 & 0 & \ldots & 0 & 0 & 0 & 2 & 0 & 0 & \ldots & 0 & 0 & 0 \\
0 & -1 & 0 & \ldots & 0 & 0 & 0 & 0 & 2 & 0 & \ldots & 0 & 0 & 0 \\
0 & 0 & -1 & \ldots & 0 & 0 & 0 & 0 & 0 & 2 & \ldots & 0 & 0 & 0 \\
\vdots & \vdots & \vdots & \ddots & \vdots & \vdots & \vdots & \vdots & \vdots & \vdots & \ddots & \vdots & \vdots & \vdots \\
0 & 0 & 0 & \ldots & -1 & 0 & 0 & 0 & 0 & 0 & \ldots & 2 & 0 & 0 \\
0 & 0 & 0 & \ldots & 0 & -1 & 0 & 0 & 0 & 0 & \ldots & 0 & 2 & 0 \\
0 & 0 & 0 & \ldots & 0 & 0 & -1 & 0 & 0 & 0 & \ldots & 0 & 0 & 2
\end{array}\right)_{2 n \times 2 n}
$$

By using Lemma 4, we obtain

$$
\begin{aligned}
& \tau\left(R\left(S_{n}\right)\right)=\operatorname{det}(P) \cdot \operatorname{det}\left(S-R P^{-1} Q\right)
\end{aligned}
$$

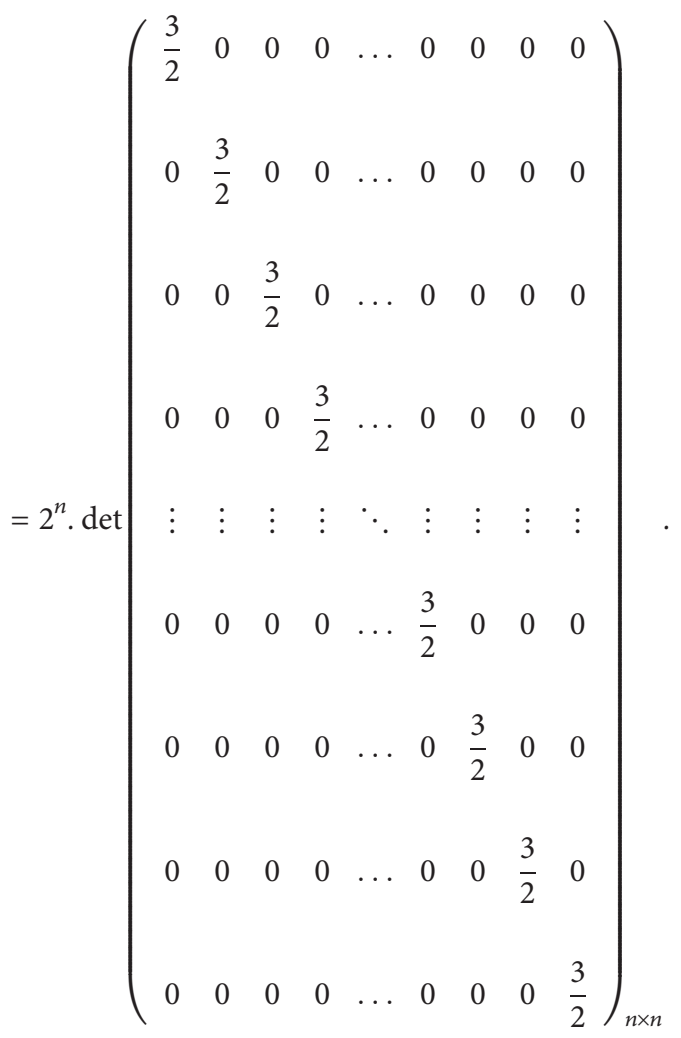

\section{Using Lemma 2,}

$$
\begin{aligned}
& =2^{n}\left(\frac{3}{2}\right)^{n} \\
& \Rightarrow \tau\left(R\left(S_{n}\right)\right)=3^{n} .
\end{aligned}
$$

Definition 6. The edge semitotal network $Q(\Gamma)$ of the network $\Gamma$ is obtained by inserting a vertex on each edge of $\Gamma$ and then by joining those new vertices that share common edges in the network $\Gamma$.

Theorem 10. For all $n$, the complexity of the edge-semitotal network $Q\left(S_{n}\right)$ is given as

$$
\tau\left(Q\left(S_{n}\right)\right)=(-1)^{n+1}(-n-1)^{n-1} .
$$

Proof. Consider the network $Q\left(S_{n}\right)$ with $\left|V\left(Q\left(S_{n}\right)\right)\right|=2 n+$ 1 and $\left|E\left(Q\left(S_{n}\right)\right)\right|=2 n+n_{2}$, see the general form in Figure 10.

Applying Lemma 1, we have

$$
\tau\left(Q\left(S_{n}\right)\right)=\frac{1}{(2 n+1)^{2}} \operatorname{det}[(2 n+1) I-\bar{D}+\bar{A}],
$$

where $\bar{D}$ and $\bar{A}$ represent the degree and adjacency matrices of $Q\left(S_{n}\right)$, respectively. 


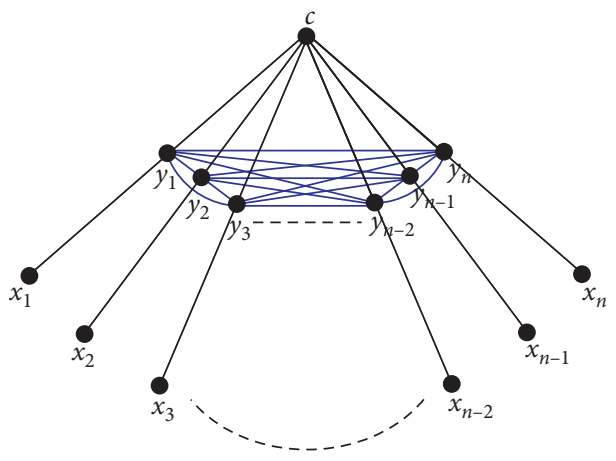

Figure 10: The edge-semitotal network $Q\left(S_{n}\right)$.

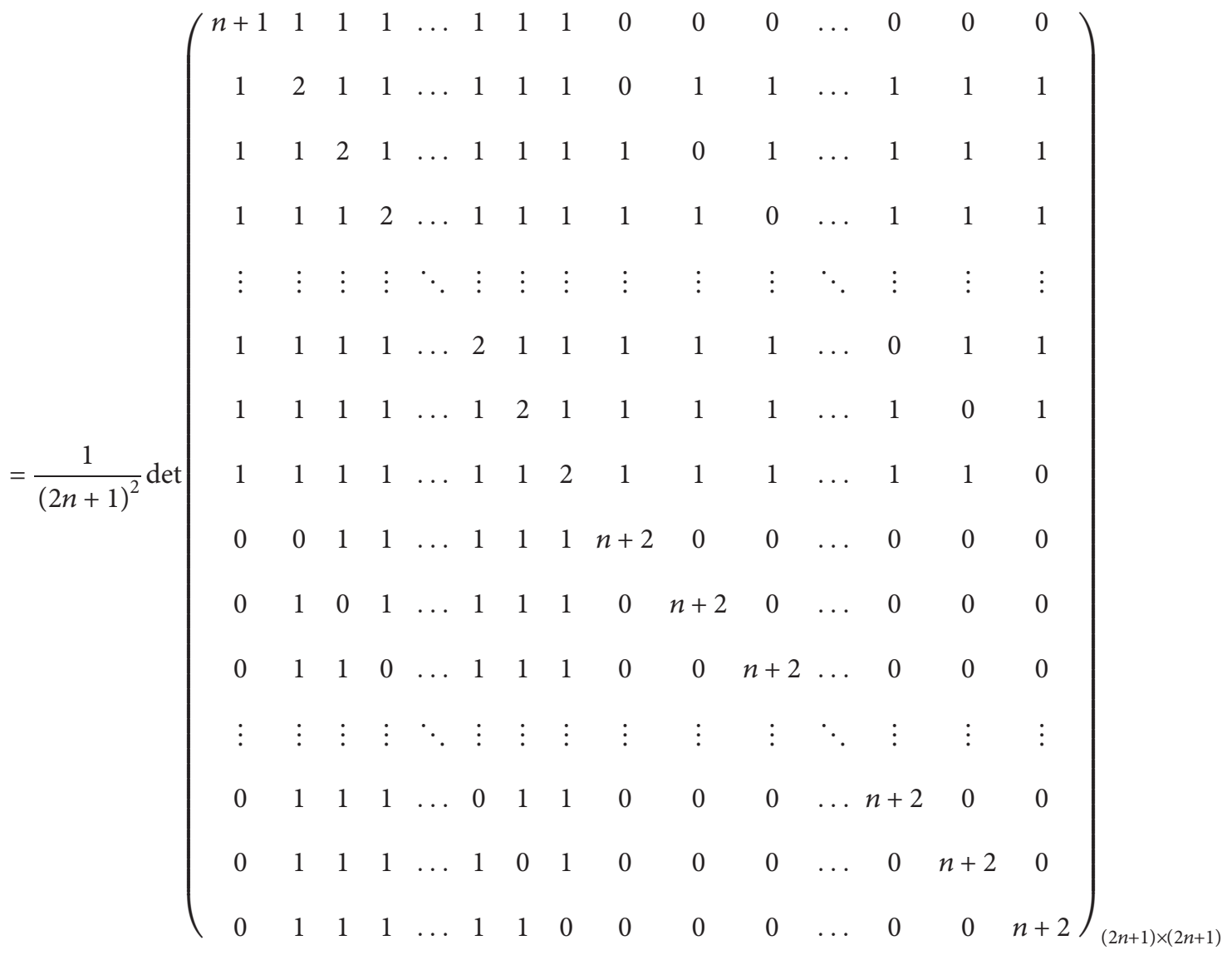




$$
\begin{aligned}
& =\operatorname{det}\left(\begin{array}{cccccccccccccc}
1 & 0 & 0 & \ldots & 0 & 0 & 0 & -1 & 0 & 0 & \ldots & 0 & 0 & 0 \\
0 & 1 & 0 & \ldots & 0 & 0 & 0 & 0 & -1 & 0 & \ldots & 0 & 0 & 0 \\
0 & 0 & 1 & \ldots & 0 & 0 & 0 & 0 & 0 & -1 & \ldots & 0 & 0 & 0 \\
\vdots & \vdots & \vdots & \ddots & \vdots & \vdots & \vdots & \vdots & \vdots & \vdots & \ddots & \vdots & \vdots & \vdots \\
0 & 0 & 0 & \ldots & 1 & 0 & 0 & 0 & 0 & 0 & \ldots & -1 & 0 & 0 \\
0 & 0 & 0 & \ldots & 0 & 1 & 0 & 0 & 0 & 0 & \ldots & 0 & -1 & 0 \\
0 & 0 & 0 & \ldots & 0 & 0 & 1 & 0 & 0 & 0 & \ldots & 0 & 0 & -1 \\
-1 & 0 & 0 & \ldots & 0 & 0 & 0 & n+1 & -1 & -1 & \ldots & -1 & -1 & -1 \\
0 & -1 & 0 & \ldots & 0 & 0 & 0 & -1 & n+1 & -1 & \ldots & -1 & -1 & -1 \\
0 & 0 & -1 & \ldots & 0 & 0 & 0 & -1 & -1 & n+1 & \ldots & -1 & -1 & -1 \\
\vdots & \vdots & \vdots & \ddots & \vdots & \vdots & \vdots & \vdots & \vdots & \vdots & \ddots & \vdots & \vdots & \vdots \\
0 & 0 & 0 & \ldots & -1 & 0 & 0 & -1 & -1 & -1 & \ldots & n+1 & -1 & -1 \\
0 & 0 & 0 & \ldots & 0 & -1 & 0 & -1 & -1 & -1 & \ldots & -1 & n+1 & -1 \\
0 & 0 & 0 & \ldots & 0 & 0 & -1 & -1 & -1 & -1 & \ldots & -1 & -1 & n+1
\end{array}\right)_{2 n \times 2 n} \\
& \Rightarrow \tau\left(Q\left(S_{n}\right)\right)=\operatorname{det}\left(\begin{array}{ll}
P_{n \times n} & Q_{n \times n} \\
R_{n \times n} & S_{n \times n}
\end{array}\right)_{2 n \times 2 n} .
\end{aligned}
$$

By using Lemma 4, we obtain

$$
\begin{aligned}
\tau\left(Q\left(S_{n}\right)\right)=\operatorname{det}(P) \cdot \operatorname{det}\left(S-R P^{-1} Q\right) & \left(\begin{array}{ccccccccc}
-n & 1 & 1 & 1 & \ldots & 1 & 1 & 1 & 1 \\
1 & -n & 1 & 1 & \ldots & 1 & 1 & 1 & 1 \\
1 & 1 & -n & 1 & \ldots & 1 & 1 & 1 & 1 \\
1 & 1 & 1 & -n & \ldots & 1 & 1 & 1 & 1 \\
\vdots & \vdots & \vdots & \vdots & \ddots & \vdots & \vdots & \vdots & \vdots \\
1 & 1 & 1 & 1 & \ldots & -n & 1 & 1 & 1 \\
1 & 1 & 1 & 1 & \ldots & 1 & -n & 1 & 1 \\
1 & 1 & 1 & 1 & \ldots & 1 & 1 & -n & 1 \\
1 & 1 & 1 & 1 & \ldots & 1 & 1 & 1 & -n
\end{array}\right)_{n \times n} .
\end{aligned}
$$

Using Lemma 2,

$$
\begin{aligned}
& =(-1)^{n}(-n+n-1)(-n-1)^{n-1} \\
& \Rightarrow \tau\left(Q\left(S_{n}\right)\right)=(-1)^{n+1}(-n-1)^{n-1} .
\end{aligned}
$$

Theorem 11. For all $n \geq 3$, the complexity of the network $\left(C_{n} \circ K_{2}\right)+K_{1}$ is given as

$$
\tau\left(\left(C_{n} \circ K_{2}\right)+K_{1}\right)=2^{n}\left[\left(\frac{7+\sqrt{33}}{4}\right)^{n}+\left(\frac{7-\sqrt{33}}{4}\right)^{n}-2\right] \text {, }
$$

where $C_{n} \circ K_{2}$ represents the rooted product of $C_{n}$ and $K_{2}$.

Proof. Consider the network $\left(C_{n} \circ K_{2}\right)+K_{1}$ with $\left|V\left(\left(C_{n} \circ K_{2}\right)+K_{1}\right)\right|=2 n+1$ and $\left|E\left(\left(C_{n} \circ K_{2}\right)+K_{1}\right)\right|=4 n$, see the general form in Figure 11.

Applying Lemma 1, we have

$$
\tau\left(\left(C_{n} \circ K_{2}\right)+K_{1}\right)=\frac{1}{(2 n+1)^{2}} \operatorname{det}[(2 n+1) I-\bar{D}+\bar{A}],
$$

where $\bar{D}$ and $\bar{A}$ represent the degree and adjacency matrices of $\left(C_{n} \circ K_{2}\right)+K_{1}$, respectively. 


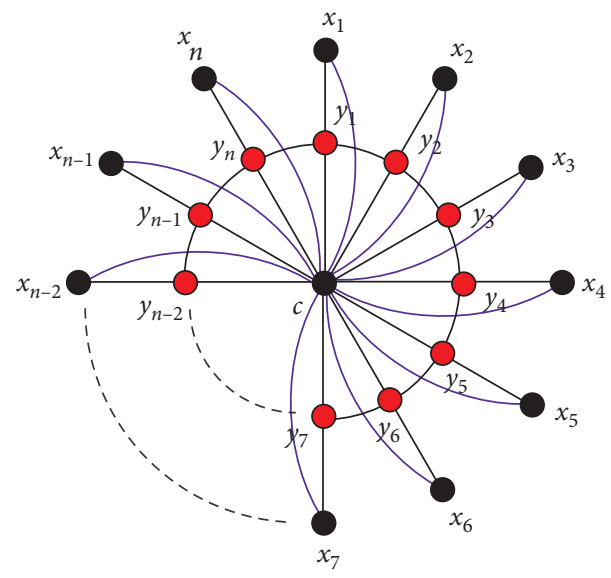

FIgURE 11: The general formation of the network $\left(C_{n} \circ K_{2}\right)+K_{1}$.

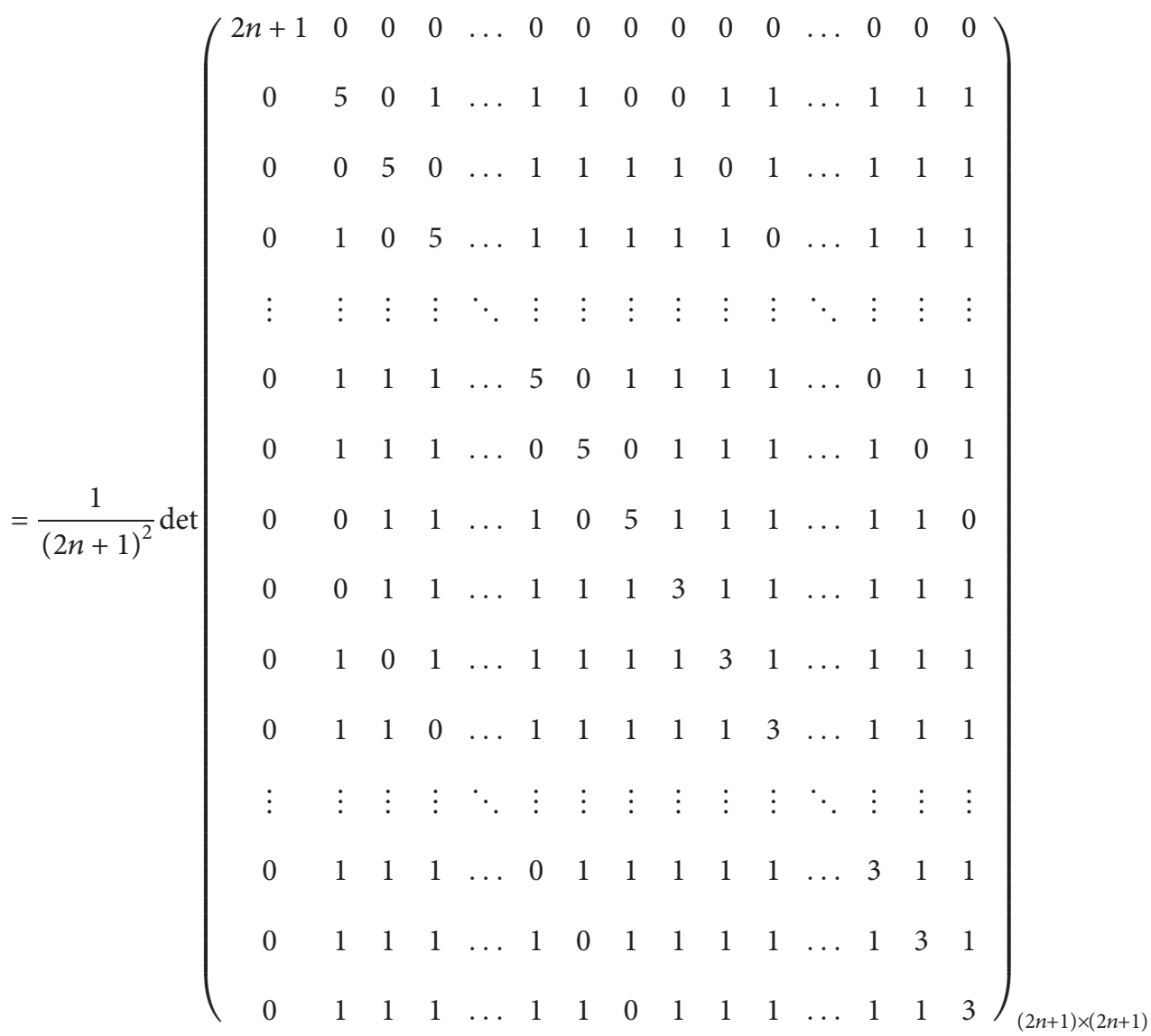




$$
=\operatorname{det}\left(\begin{array}{cccccccccccccc}
4 & -1 & 0 & \ldots & 0 & 0 & -1 & -1 & 0 & 0 & \ldots & 0 & 0 & 0 \\
-1 & 4 & -1 & \ldots & 0 & 0 & 0 & 0 & -1 & 0 & \ldots & 0 & 0 & 0 \\
0 & -1 & 4 & \ldots & 0 & 0 & 0 & 0 & 0 & -1 & \ldots & 0 & 0 & 0 \\
\vdots & \vdots & \vdots & \ddots & \vdots & \vdots & \vdots & \vdots & \vdots & \vdots & \ddots & \vdots & \vdots & \vdots \\
0 & 0 & 0 & \ldots & 4 & -1 & 0 & 0 & 0 & 0 & \ldots & -1 & 0 & 0 \\
0 & 0 & 0 & \ldots & -1 & 4 & -1 & 0 & 0 & 0 & \ldots & 0 & -1 & 0 \\
-1 & 0 & 0 & \ldots & 0 & -1 & 4 & 0 & 0 & 0 & \ldots & 0 & 0 & -1 \\
-1 & 0 & 0 & \ldots & 0 & 0 & 0 & 2 & 0 & 0 & \ldots & 0 & 0 & 0 \\
0 & -1 & 0 & \ldots & 0 & 0 & 0 & 0 & 2 & 0 & \ldots & 0 & 0 & 0 \\
0 & 0 & -1 & \ldots & 0 & 0 & 0 & 0 & 0 & 2 & \ldots & 0 & 0 & 0 \\
\vdots & \vdots & \vdots & \ddots & \vdots & \vdots & \vdots & \vdots & \vdots & \vdots & \ddots & \vdots & \vdots & \vdots \\
0 & 0 & 0 & \ldots & -1 & 0 & 0 & 0 & 0 & 0 & \ldots & 2 & 0 & 0 \\
0 & 0 & 0 & \ldots & 0 & -1 & 0 & 0 & 0 & 0 & \ldots & 0 & 2 & 0 \\
0 & 0 & 0 & \ldots & 0 & 0 & -1 & 0 & 0 & 0 & \ldots & 0 & 0 & 2
\end{array}\right)_{2 n \times 2 n}
$$

By using Lemma 4, we obtain

$$
\begin{aligned}
& \tau\left(\left(C_{n}{ }^{\circ} K_{2}\right)+K_{1}\right)=\operatorname{det}(P) \cdot \operatorname{det}\left(S-R P^{-1} Q\right)
\end{aligned}
$$

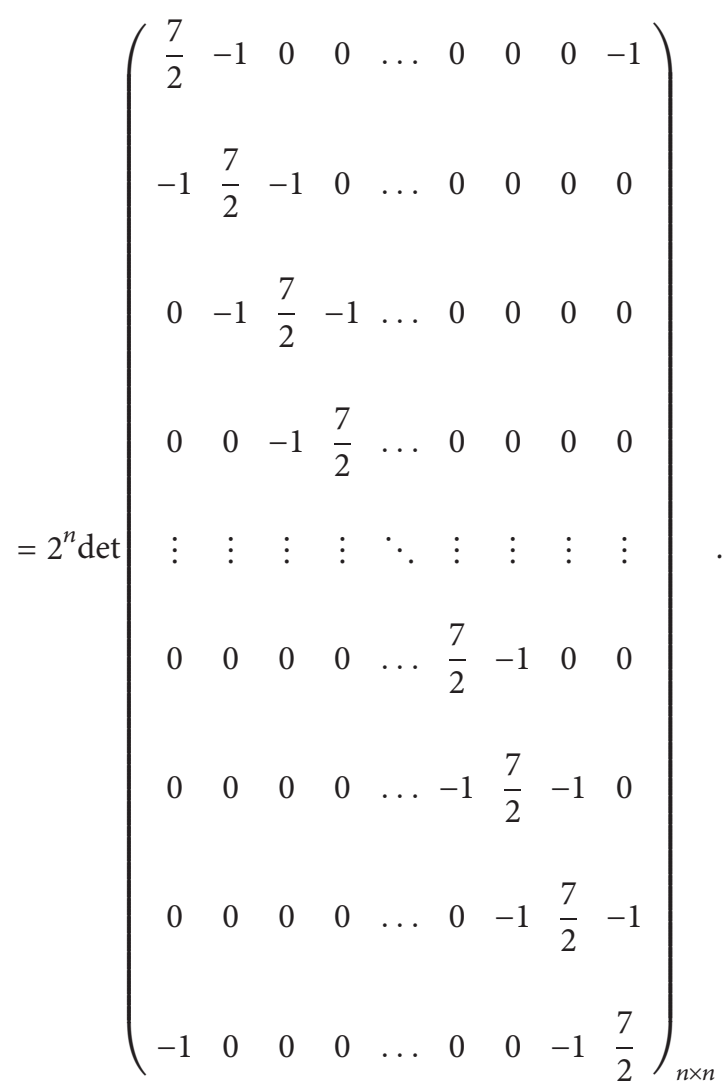


TABLE 1: Synopsis of the main findings.

\begin{tabular}{lccc}
\hline Network & Parameters & Complexity & Planar vs. nonplanar \\
\hline$S_{n}+W_{3}$ & $\forall n \in \mathbb{N}$ & $5^{n-1}(n+5)^{4}$ & Nonplanar \\
$D_{2}\left(S_{n}\right)$ & $\forall n \in \mathbb{N}$ & $n \cdot 2^{2 n}$ & Planar \\
$S^{\prime}\left(S_{n}\right)$ & $\forall n \in \mathbb{N}$ & $n \cdot 2^{n-1}$ & Planar \\
$S_{n} \otimes P_{2}$ & $\forall n \in \mathbb{N}$ & $8^{n}(n+1)$ & Planar \\
$V^{\prime}\left(S_{n}\right)$ & $\forall n \in \mathbb{N}$ & $n^{n}(n+1)^{n-1}$ & Nonplanar \\
$S_{n}+K_{2}$ & $\forall n \in \mathbb{N}$ & $3^{n-2}\left[(2 n+6)^{2}-(n+3)^{2}\right]$ & Planar \\
$W_{n}^{\prime}$ & $n \geq 3$ & $2^{2 n}-2^{n+1}+1$ & Planar \\
$W_{n} \circ P_{2}$ & $n \geq 3$ & $((3+\sqrt{5}) / 2)^{n}+((3-\sqrt{5}) / 2)^{n}-2$ & Planar \\
$R\left(S_{n}\right)$ & $\forall n \in \mathbb{N}$ & $3^{n}$ & Planar \\
$Q\left(S_{n}\right)$ & $\forall n \in \mathbb{N}$ & $(-1)^{n+1}(-n-1)^{n-1}$ & Nonplanar \\
$\left(C_{n} \circ P_{2}\right)+K_{1}$ & $\forall n \in \mathbb{N}$ & $2^{n}\left[((7+\sqrt{33}) / 4)^{n}+((7-\sqrt{33}) / 4)^{n}-2\right]$ & Nonplanar \\
\hline
\end{tabular}

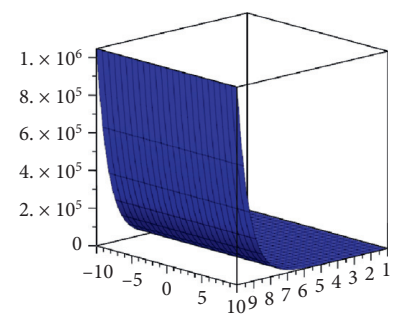

(a)

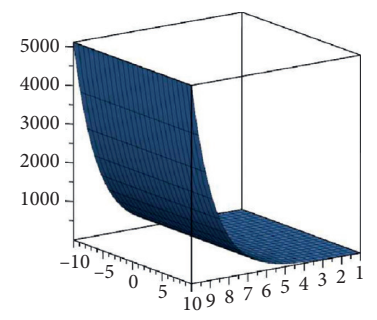

(e)

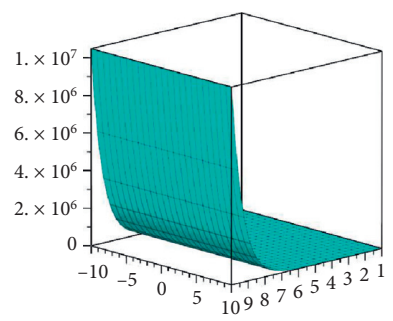

(b)

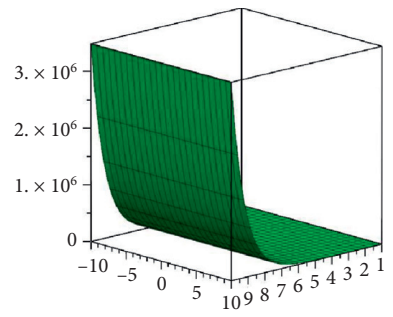

(f)

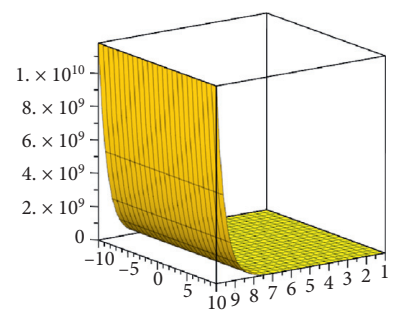

(c)

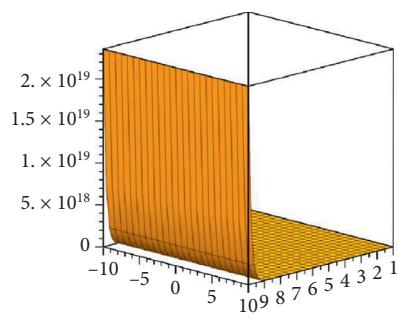

(g)

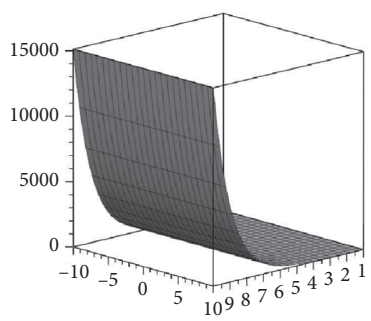

(d)

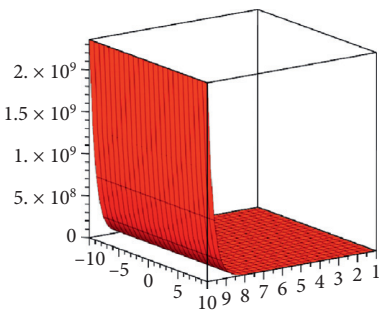

(h)

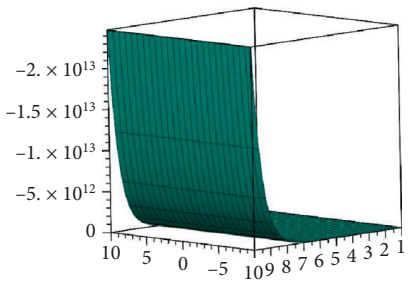

(i)

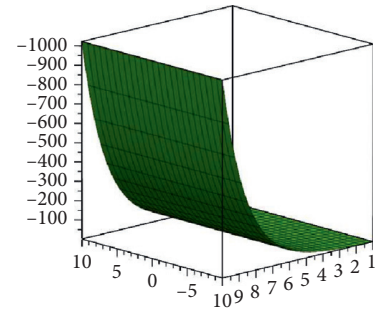

(j)

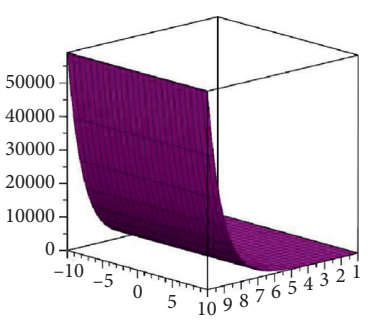

(k)

Figure 12: Graphical behaviors of the complexities of the networks $W_{n}^{\prime} \longrightarrow(\mathrm{a}), D_{2}\left(S_{n}\right) \longrightarrow(\mathrm{b}), S_{n} \otimes P_{2} \longrightarrow(\mathrm{c}), W_{n} \circ P_{2} \longrightarrow(\mathrm{d}), Q\left(S_{n}\right) \longrightarrow(\mathrm{e})$, $S_{n}+K_{2} \longrightarrow(\mathrm{f}), V^{\prime}\left(S_{n}\right) \longrightarrow(\mathrm{g}), S^{\prime}\left(S_{n}\right) \longrightarrow(\mathrm{h}), S_{n}+W_{3} \longrightarrow(\mathrm{i}), R\left(S_{n}\right) \longrightarrow(\mathrm{j})$, and $\left(C_{n} \circ P_{2}\right)+K_{1} \longrightarrow(\mathrm{k})$.

Using Lemma 2,

$$
\begin{aligned}
& =2^{n} \cdot 2\left[T_{n}\left(\frac{7}{4}\right)-1\right] \\
& \Rightarrow \tau\left(\left(C_{n} \circ K_{2}\right)+K_{1}\right)=2^{n}\left[\left(\frac{7+\sqrt{33}}{4}\right)^{n}+\left(\frac{7-\sqrt{33}}{4}\right)^{n}-2\right] .
\end{aligned}
$$

\section{Synopsis and 3D Comparison of the Complexities of the Networks}

This section comprises a tabular summary and 3D graphical plots and comparison of the closed formulae for the complexities of networks calculated in this article :

(i) Table 1 indicates a brief summary of our findings in the form of complexity of various networks and also 


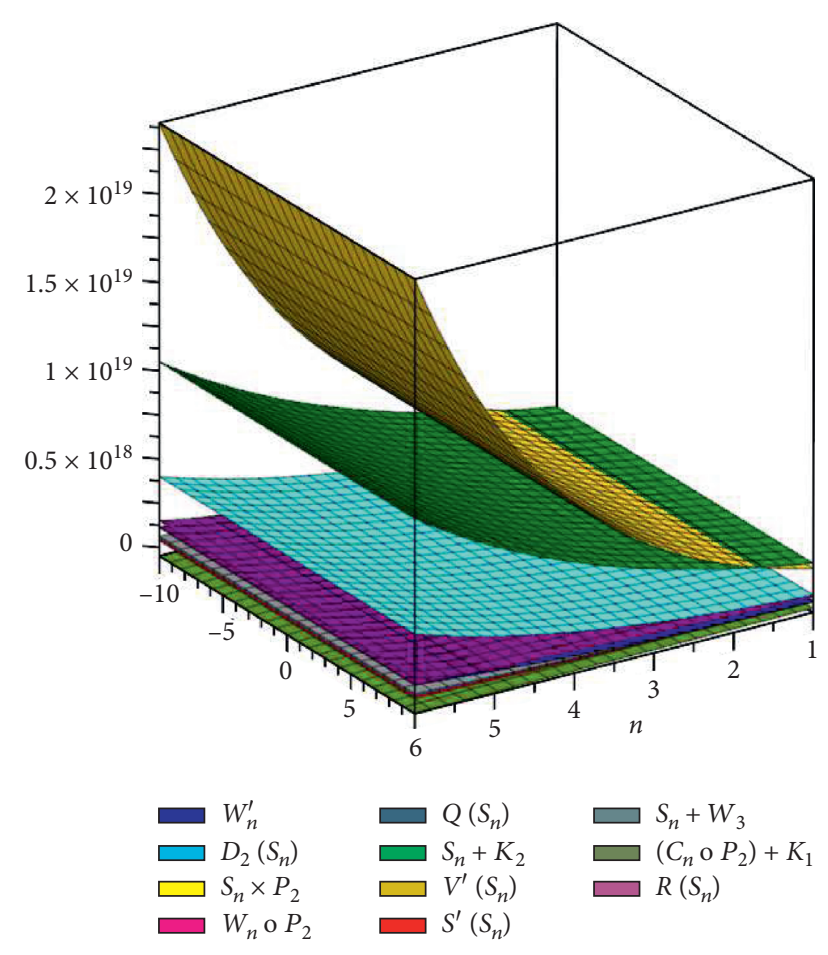

FIgURE 13: Graphical comparison of the complexities of the networks.

categorizes the network being planar or nonplanar

(ii) Figure 12 shows the individual 3D graphical tends of the values of the complexity of networks discussed in this article, whereas Figure 13 reveals the relative comparison of the values of the complexity of these networks, reveling the golden one to be the dominated layer among all

\section{Conclusion}

The complexity, i.e., number of spanning trees, of a network is a purposeful algebraic invariant. The calculation of this measurement gives an important information about the reliability of a network by providing the information of total number of acyclic networks present within. In this article, we have computed the complexity of networks operation such as $W_{n}^{\prime}, D_{2}\left(S_{n}\right), S_{n} \otimes P_{2}, W_{n} \circ P_{2}, Q\left(S_{n}\right), S_{n}+K_{2}, V^{\prime}\left(S_{n}\right)$, $S^{\prime}\left(S_{n}\right), S_{n}+W_{3}, R\left(S_{n}\right)$, and $\left(C_{n} \circ P_{2}\right)+K_{1}$. Mainly, our techniques have been algebraic and involve Chebyshev polynomials and concepts of the matrix theory while calculating our results.

\section{Data Availability}

All the data are included within this paper. However, more details of the data are available from the corresponding author upon request.

\section{Conflicts of Interest}

The authors declare that they have no conflicts of interest.

\section{References}

[1] N. L. Biggs, Algebraic Graph Theory, Cambridge University Press, Cambridge, UK, 2 edition, 1993.

[2] D. Cvetkovic, M. Doob, and H. Sachs, Spectra of Graphs, Academic Press, New York, NY, USA, 1978.

[3] F. Harary, Graph Theory, Addison-Wesley, Reading, MA, USA, 1969.

[4] G. G. Kirchhoff, "Uber die Auflosung der Gleichungen, auf welche man beider Untersuchung der Linearen Verteilung Galvanischer Strme gefhrt wird," Annual Review of Physical Chemistry, vol. 72, pp. 497-508, 1847.

[5] H. N. V. Temperley, "Combinatorics," in Proceedings on Combinatorial Mathematics, pp. 356-357, Mathematics Institute, Oxford, UK, 1972.

[6] D. J. A. Welsh, "Complexity: Knots, Colourings and Counting," London Mathematical Society Lecture Notes Series 186, Cambridge University Press, Cambridge, UK, 1993.

[7] G. A. Cayley, "A theorem on trees," The Quarterly Journal of Mathematics, vol. 23, pp. 276-378, 1889.

[8] J. Sedlacek, "On the skeleton of a graph or digraph," in Combinatorial Structures and Their Applications, M. Guy, N. Saver Hanani, and J. Schonheim, Eds., pp. 387-391, Gordon and Breach, New York, NY, USA, 1970.

[9] S. N. Daoud, "The deletion-contraction method for counting the number of spanning trees of graphs," The European Physical Journal Plus, vol. 130, pp. 1-14, 2015.

[10] A. K. Kelmans and V. M. Chelnokov, "A certain polynomials of a graph and graphs with an extermal number of trees," Journal of Combinatorial Theory, Series B, vol. 16, pp. 197-214, 1974.

[11] S. N. Daoud, "Number of spanning trees for shadow of some graphs," International Journal of Mathematical Combinatorics, vol. 4, pp. 103-115, 2013.

[12] S. N. Daoud, "Generating formulas of the number of spanning trees of some special graphs," The European Physical Journal Plus, vol. 129, pp. 1-14, 2014.

[13] S. N. Daoud, "Number of spanning trees of different products of complete and complete bipartite graphs," The Journal Mathematical Problems in Engineering, vol. 2014, Article ID 965105, 23 pages, 2014.

[14] S. N. Daoud and K. Mohamed, "The complexity of some families of cycle-related graphs," Journal of Taibah University for Science, vol. 11, no. 2, pp. 205-228, 2017.

[15] S. N. Daoud, "Number of spanning trees of cartesian and composition products of graphs and Chebyshev polynomials," IEEE Access, vol. 7, pp. 71142-71157, 2019.

[16] S. N. Daoud, "Some applications of number of spanning trees of complete and complete bipartite graphs," American Journal of Applied Sciences, vol. 9, no. 4, pp. 584-592, 2012.

[17] J. C. Mason and D. C. Handscomb, Chebyshev Polynomials, Chapman and Hall/CRC, Boca Raton, FL, USA, 2003.

[18] S. N. Daoud, "Complexity of products of some complete and complete bipartite graphs," Journal of Applied Mathametics, vol. 2013, Article ID 673270, 25 pages, 2013.

[19] D. J. Gross, T. J. Saccoman, and L. C. Suffel, Spanning Tree Results for Graphs and Multigraphs: A Matrix-Theoretic Approach, World Scientific Publishing Company, Singapore, 2014.

[20] A. Bose and K. Saha, Random Circulant Matrices, CRC Press, Taylor and Francis Group, Boca Raton, FL, USA, 2019.

[21] M. Marcus, A Servy of Matrix Theory and Matrix Inequalities, University of Allyn and Bacon Inc., Boston, MA, USA, 1964. 
[22] R. Balakrishnan and K. Ranganathan, A Textbook of Graph Theory, Springer, New York, NY, USA, 2000.

[23] D. B. West, Introduction to Graph Theory, Prentice- Hall of India, New Delhi, India, 2 edition, 2003. 